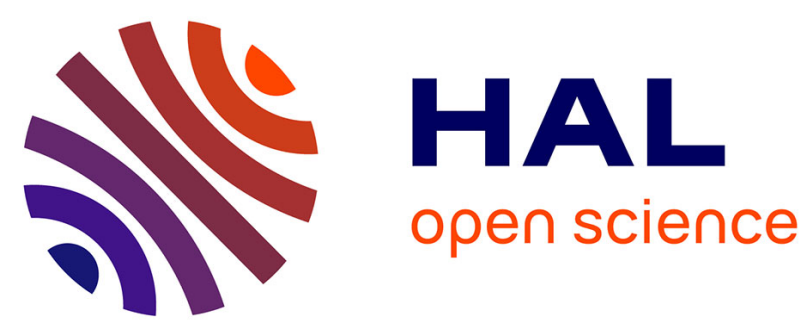

\title{
Laue microdiffraction at the ESRF
}

Odile Robach, Christoph Kirchlechner, Jean Sébastien Micha, M. Olivier Ulrich, Xavier Biquard, Olivier Geaymond, Olivier Castelnau, Michel Bornert, Johann Petit, Sophie Berveiller, et al.

\section{- To cite this version:}

Odile Robach, Christoph Kirchlechner, Jean Sébastien Micha, M. Olivier Ulrich, Xavier Biquard, et al.. Laue microdiffraction at the ESRF. Strain and Dislocation Gradients from Diffraction: SpatiallyResolved Local Structure and Defects, Imperial College Press, pp.156-204, 2014, 978-1-908979-63-6. 10.1142/9781908979636_0005. hal-02161265

\section{HAL Id: hal-02161265 https://hal.science/hal-02161265}

Submitted on 20 Jun 2019

HAL is a multi-disciplinary open access archive for the deposit and dissemination of scientific research documents, whether they are published or not. The documents may come from teaching and research institutions in France or abroad, or from public or private research centers.
L'archive ouverte pluridisciplinaire HAL, est destinée au dépôt et à la diffusion de documents scientifiques de niveau recherche, publiés ou non, émanant des établissements d'enseignement et de recherche français ou étrangers, des laboratoires publics ou privés. 


\section{Laue Microdiffraction at the ESRF}

O. Robach ${ }^{\mathrm{a}, \mathrm{d}}$, C. Kirchlechner ${ }^{\mathrm{f}, \mathrm{g}}$, J.S. Micha ${ }^{\mathrm{b}, \mathrm{d}}$, O. Ulrich ${ }^{\mathrm{a}, \mathrm{d}}$, X. Biquard ${ }^{\mathrm{a}, \mathrm{d}}$, O. Geaymond ${ }^{\mathrm{e}, \mathrm{d}}$, O. Castelnau ${ }^{\mathrm{h}}$, M. Bornert $^{\mathrm{i}}$, J. Petit ${ }^{\mathrm{h}, \mathrm{j}}$, S. Berveiller ${ }^{\mathrm{m}}$, O. Sicardy ${ }^{\mathrm{k}}$,

J. Villanova ${ }^{1}$ and F. Rieutord ${ }^{\mathrm{a}, \mathrm{d}}$

${ }^{a} C E A-G r e n o b l e, ~ I N A C / S P 2 M / N R S$,

${ }^{\mathrm{b}} U M R$ SPrAM CNRS-CEA-UJF, CEA-Grenoble, INAC,

${ }^{\mathrm{c}}$ CEA-LETI, MINATEC Campus,

17 rue des Martyrs, 38054 Grenoble Cedex 9, France

${ }^{\mathrm{d}}$ CEA-CNRS CRG-IF BM32 beamline at ESRF,

6 rue Jules Horowitz,

38043 Grenoble Cedex 9,France

e InstitutNéel, CNRS 25 rue des Martyrs,

F-38042 Grenoble, France

${ }^{\mathrm{f}}$ University of Leoben, Jahnstr. 12, A-8700 Leoben, Austria,

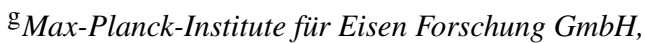

Max-Planck-Straze 1, 40237, Düsseldorf, Germany

h PIMM, CNRS, 151 Bd de l'Hôpital, 75013 Paris

${ }^{\mathrm{i}}$ Laboratoire NAVIER, Université Paris-Est, École des ponts ParisTech,

77455 Marne-la-Vallée, France

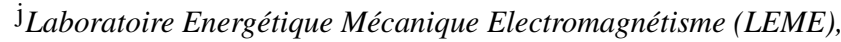
EA4416, Université Paris Ouest, 92410 Ville d'Avray, France

${ }^{\mathrm{k}}$ CEA, LITEN, F-38054 Grenoble, France

${ }^{1}$ ESRF, 6 rue Jules Horowitz, 38043 Grenoble Cedex 9,

${ }^{\mathrm{m}}$ Laboratoire d'Etude des Microstructures et de Mécanique des Matériaux (LEM3), Arts et Métiers ParisTech,

4, rue Augustin Fresnel, 57078 Metz, France 


\begin{abstract}
This chapter describes the Laue microdiffraction station of the French CEACNRS CRG-IF BM32 beamline at the European Synchrotron Radiation Facility (ESRF), with its available special methods and planned upgrades. Applications are discussed, with an overview of scientific questions addressed by user. Three examples of user studies are presented. Two of them, concerning shape memory alloys and solid oxide fuel cells, are only briefly described. The third example, concerning compressive and tensile tests on single-crystalline micropillars, is described more extensively. Here, the data from two papers are examined in an attempt to i) separate intrinsic effects (e.g. size effects) from extrinsic effects (due to non-ideal boundary conditions at the ends of the pillars), and ii) include in the description the crystallographic orientation of the pillar-free surfaces, in order to find the best conditions for detecting edge dislocations of the most activated glide system possibly piled up against a surface barrier.
\end{abstract}

\title{
5.1. Introduction and Context
}

The Laue microdiffraction instrument of the CEA-CNRS BM32 beamline at ESRF was installed in 2006, taking advantage of an upgrade of the optics of the beamline, initially constructed in 1994. This upgrade has allowed the setup of a new white X-ray beam operation mode (5-30 keV) in addition to the existing "monochromatic mode" (Baudoing-Savois et al., 1999; Ulrich et al., 2011). The instrument shares the beamline with two other instruments which are dedicated to surface and interface science, and offer a panel of diffraction and scattering techniques (surface diffraction, reflectivity, glancing incidence X-ray scattering, under ultrahigh vacuum (UHV) or in air). The amount of beamtime dedicated to Laue microdiffraction is around $25 \%$. The construction of the setup has been motivated by the needs of the microelectronics industry in terms of "in volume" stress measurements in polycrystalline layers ill-adapted for classical methods (too many grains for single-crystal diffraction and not enough for powder diffraction), e.g. in copper interconnects. The new setup allows a fast and accurate two-dimensional (2D) mapping of the local crystalline orientations and elastic stresses (for the deviatoric part) at the micron scale in polycrystals. Three-dimensional (3D) space-resolution (along the incident X-ray beam) or access to the "hydrostatic" part of the stress is also available.

This chapter is organized as follows. Section 5.2 describes the current status of the setup and the planned upgrades (hardware, methods and software). Section 5.3 lists a series of examples of application, briefly 
describing two of them. Section 5.4 focuses on one particular application (Kirchlechner et al., 2011a, 2011b), the in situ study of single-crystalline micropillars during mechanical tests. The chapter concludes with a consideration of optimal geometry for cutting micropillars and for choosing their orientation with respect to the incident beam, the setup's future possibilities and a number of open-ended questions.

\subsection{Experimental Details on BM32}

\subsubsection{Beamline layout (beam production)}

\subsubsection{Optics hutch}

The X-ray source is a bending magnet with a critical energy of $19.8 \mathrm{keV}$, providing a flat photon flux spectrum in the range of 5-25 keV used for white beam microdiffraction. A monochromatic macro beam $\left(200 \times 500 \mu \mathrm{m}^{2}\right)$ is used by the other two instruments, while a focused white beam is delivered to the microdiffraction setup. The beam from the source is first focused on variable-aperture microslits $\left(20 \times 20 \mu \mathrm{m}^{2}\right)$ at the exit of the optics hutch, which acts as a fixed secondary source for the experimental hutch downstream, then refocused on the sample in the experimental hutch down to $0.5 \times 0.7$ microns $^{2}$ using Kirkpatrick-Baez $(\mathrm{K}-\mathrm{B})$ mirrors. In the whitebeam mode the primary optics features two mirrors. Both mirrors contribute to focusing the beam in the vertical plane on the microslit. Additionally, they both act as a low-bandpass energy filter with a cut-off energy, which is adjustable by the inclination of the mirrors (from 13 to $25 \mathrm{keV}$ ). In the monochromatic-beam mode (resolution $\mathrm{dE} / \mathrm{E}=1.10^{-4}$ ), the doublecrystal monochromator is inserted between the two mirrors. The initial vertical position of the beam on the microslit is retained by reducing the mirror's inclination. The beam is focused in the horizontal direction using sagittal bending of the second crystal of the monochromator to optimize the flux on the sample.

\subsubsection{Experimental hutch}

A set of two K-B mirrors with adjustable curvature is used to achromatically focus the beam in two directions. The reflection geometry of the experiment uses a $40^{\circ}$ incidence angle $\theta$ of the beam on the sample (with respect to the 
surface) and a $90^{\circ}$ average $2 \theta$ scattering angle. The cone of collection of the diffracted beams by the $2 \mathrm{D}$ detector has a maximum opening of $100^{\circ}$. The incident divergence is of the order of $1 \mathrm{mrad}$ in the two directions. For the usual sample-detector working distance, the angular opening of a pixel of the detector is also of the order of $1 \mathrm{mrad}$. As a consequence, a variation of $10^{-4}$ on the orientation or the deviatoric strains of the unit cell of a grain induces variations in the spot positions of the order of 0.1 pixel. As the setup shares the hutch with one of the diffractometers, it is easy to transport and realign.

\subsubsection{Beam features}

A beamsize of $0.8 \times 0.9 \mu \mathrm{m}^{2}$ (horizontal $\times$ vertical) projected onto the sample surface $\left(50^{\circ}\right.$ off the vertical) is routinely obtained for white beam. This size is stable over several days in the horizontal direction, and can be readjusted every 12 hours in the vertical direction by running an automatic procedure lasting a few minutes. The calculated photon flux on the sample is of the order of $10^{10} \mathrm{ph} / \mathrm{s}$ in the white-beam mode. The maximum pixel intensity of a (531) spot of a Ge single crystal at $11.9 \mathrm{keV}$ decreases by a factor of ten between the white and monochromatic beam modes.

\subsubsection{Detectors - sample environment - accessories}

Figure 5.1 shows a photograph of the setup.

For the measurement of Laue patterns, three charge-coupled device (CCD) cameras with fluorescent screens and a demagnification by opticalfiber taper are available. Two have a high sensitivity: a MAR Research (now Rayonix) camera (165 mm diameter, 80 microns pixel size, 5 second readout time) and a Roper, with the same geometry, offering a faster readout ( 2.35 seconds). The photonics Science VHR $(2671 \times 4008$ pixels screen size, 31 microns pixel size) is faster than the other two cameras but less sensitive, due to a smaller fill factor that allows a pipeline mode (exposing and reading at the same time) with an effective zero readout time for exposure times larger than one second.

An energy-resolved silicon drift detector (SDD) with a Multi Channel Analyzed (MCA) card (RontecXFlash) is available for fluorescence 


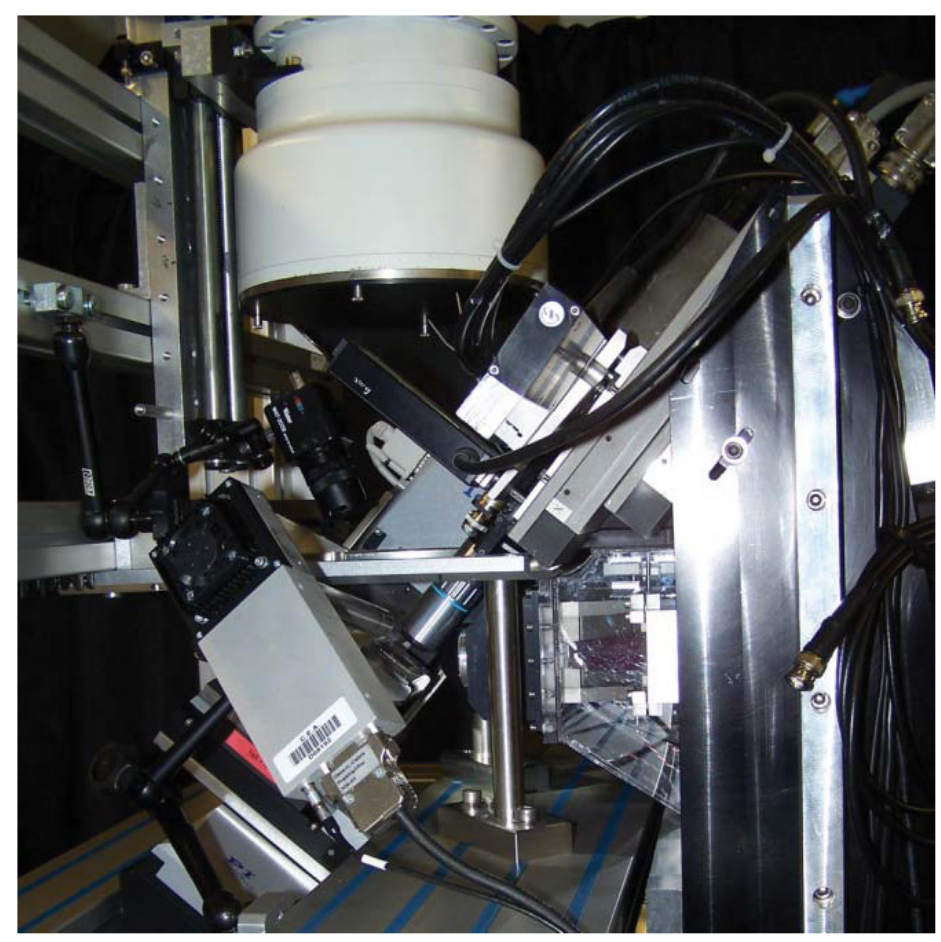

Fig. 5.1. Photograph of the Laue microdiffraction setup showing the different elements ("alignment" configuration with microscope in position and retracted detector) (Ulrich et al., 2011).

measurements during beam alignment, beamsize optimization and regionof-interest localization of samples. It can also be mounted on two translation stages to determine the photon energies of diffraction spots (not intercepted by the $2 \mathrm{D}$ detector) to access the non-deviatoric part of the strain tensor of the unit cell (Robach et al., 2011). The sample is mounted on a xyz translation stage of $25 \mathrm{~mm}$ range and 0.1 micron minimum step in the three directions.

The space around the sample can host devices for mechanical testing on macro (e.g. DEBEN $5000 \mathrm{~N}$ ) and micro (Kirchlechner et al., 2011c) samples, controlled-atmosphere ovens (a commercial oven manufactured by Anton Parr is available for users), chemical etching cells and a cooling gun for radiation-sensitive samples. An optical microscope (field $20 \times$ $30 \mu \mathrm{m}^{2}-200 \times 300 \mu \mathrm{m}^{2}$ with continuous zoom) is used to visualize the 
surface of the sample during the alignment phase. The focal distance of the microscope serves as a reference to make the sample surface cross the incident beam at the same position as the reference sample used to calibrate the detection geometry. The microscope is later retracted to give way to the $2 \mathrm{D}$ detector.

\subsubsection{Software}

\subsubsection{Control command and online data analysis}

Data acquisition (motors, detectors) is controlled through the SPEC software (http://www.certif.com) and the data is later displayed on various interfaces (e.g. Newplot [http://www.esrf.eu], PyMCA [http://pymca. sourceforge.net] and Imagej [http://rsbweb.nih.gov/ij/]). The sample holder usually supports two additional specimens that can be brought into the $\mathrm{X}$-ray beam during the experimental runs or alignment procedure. The Laue pattern of an unstrained Ge single crystal is used to calibrate the experimental geometry (detector position and tilt angles with respect to the probe volume and beam direction), and a small area of gold $\left(40 \times 100 \mu \mathrm{m}^{2}\right)$ deposited on a silicon substrate allows the periodical checking of the size and position of the X-ray beam in both directions (through the fluorescence emission yield).

Routines for automatic re-optimization of the beamsize using the gold rectangle are available if necessary during the measurements. The optimal setup of the $\mathrm{K}-\mathrm{B}$ focusing is achieved by minimizing the beam position spread obtained when scanning a $10 \mu \mathrm{m}$ slit located upstream of the mirrors to select different rays (the so-called "wave-fronts method") (Hignette et al., 2005). Beamsize and position can be monitored using the microscope and a $5 \mu \mathrm{m}$-thick fluorescent yttrium aluminum garnet (YAG) crystal. The region of interest in a specimen can be easily memorized to allow further investigations after realignment procedures. At the beginning of each experiment two terabytes are available to store recorded images (8 or 19 megabytes/image depending on the detector). Software tools for monitoring a given diffraction spot on the $2 \mathrm{D}$ detector as a function of sample position (report of intensity, pixel position, generation of mosaic image) are available for accurate sample positioning when combining polyand monochromatic measurements. 


\subsubsection{Data analysis (offline data analysis)}

An Open Source software package called LaueTools has been developed (http://sourceforge.net/projects/lauetools; Micha and Robach, 2013) for the analysis of the Laue patterns. It reproduces in part the functionalities of the X-ray Microdiffraction Analysis Software (XMAS) of the Advanced Light Source (ALS) (http://xraysweb.lbl.gov/microdif/user_resources.htm) (Chung and Ice, 1999; Tamura et al., 2003). Graphical interfaces and Python scripts allow for great flexibility in the definition of the analysis sequence. This is particularly useful in improving the analysis of a series of images without having to restart from the beginning.

The analysis of a Laue pattern proceeds in two stages. The first is the calibration of the experimental geometry using the Ge Laue pattern: peak search, manual pre-adjustment of the crystal orientation and geometry parameters by visual matching between the theoretical and experimental patterns, then automatic refining of the orientation and the geometry. This analysis can be automated once the geometrical parameters are approximated.

The second stage, which can be automated on a series of images (e.g. for maps), is the treatment of the local Laue pattern of the real sample: peak search, elimination of spots with asymmetric shapes, automatic multigrain indexation and, for each grain refinement of the strained orientation matrix (orientation + deviatoric strain tensor), possibly removing the spots which deviate too much from their theoretical position (e.g. by one pixel).

A module for simulating Laue patterns in the presence of various crystal defects in the analysis volume (twins, strain/orientation distribution) is also available.

\subsection{4. “Add-on" methods}

\subsubsection{Differential Aperture X-ray Microscopy (DAXM)}

In 2010, a wire-scanning system for DAXM (Larson et al., 2002) was added to obtain spatial resolution around 1 micron along the incident beam by triangulation (Richard, 2012). Figure 5.2 shows a photograph of the wire-scanning setup and a diagram of the geometry.

The position of a pixel on the camera and the position of the wire when it starts masking the signal of this pixel define a geometric plane. The 


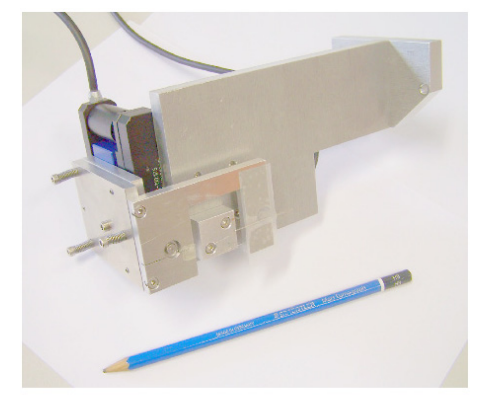

(a)

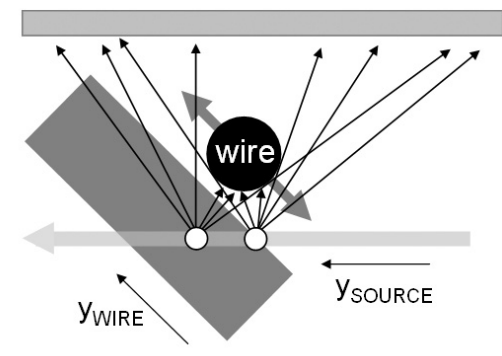

(b)

Fig. 5.2. Wire-scanning setup. (a) Picture, this part is mounted on the sample translation stages during the experiments. The fork holding the wire is mounted on a $(y, z)$ stage (range z $10 \mathrm{~mm}$, y $15 \mathrm{~mm}$ ). (b) Geometry, the $\mathrm{W}$ wire $(50 \mu \mathrm{m}$ diameter, $300 \mu \mathrm{m}$ above the surface) travels along the yWIRE and successively blocks the diffracted rays coming from different depths in the sample. A pseudo-motor allows the wire to move with constant steps of the wire shadow on the camera. The sample-detector distance (not to scale) is $70 \mathrm{~mm}$.

intersection of this plane with the incident beam defines the position of the diffracting volume producing this signal. For each xy beam position on the sample, 400 images at different wire positions are recorded to obtain 50 "depth-resolved" images. The software package for the reconstruction of the depth-resolved images from the raw images of the wire scan is under development. The procedure will include a stage of characterizing the real position of the wire in the detector frame, using a wire scan on the Ge.

\subsubsection{Monochromatic mode (7-22 keV)}

Successive white and monochromatic beams on the same point of a grain in the sample allow six lattice parameters to be obtained, and therefore the full local stress tensor, including the hydrostatic part, via the measurement of the photon energy of one or several spots. A prior analysis of the Laue pattern collected with white beam provides the energy (calculated with a hypothetic lattice parameter) to be used in the monochromatic mode to observe a certain (hkl) spot of the grain. The energy of the monochromatic beam is then scanned to find the exact spot energy. The $-\mathrm{dE} / \mathrm{E}$ between theory and experiment then provides the lattice expansion $(\mathrm{dV} / \mathrm{V})_{1 / 3}$. At each experiment, the energies of at least three spots of the Ge single crystal 
are measured to calibrate the change in orientation of the incident beam (with respect to the samples) between the two modes (around $0.3 \mathrm{mrad}$ ). The online analysis of the energy scans allows the checking of the energy profiles for shape and reproducibility.

\subsubsection{Measurements of the full strain tensor: white-beam method}

Since 2009, the photon energy of the diffraction spots can also be measured in the white-beam mode, by using the energy-resolved detector. As for the monochromatic method, a prior analysis of the Laue pattern is necessary, but this time so as to predict the position of the yz translation stages of the point detector to detect a certain (hkl) spot of the grain. The calculation of the spot energies allows the selection of spots that are far away in energy from the fluorescence lines of the sample. Figure 5.3 shows the energy spectrum collected on a diffraction spot of a $\mathrm{UO}_{2}$ micrograin.

This method allows the simultaneous measurement of the Laue pattern and the energy of one spot, ensuring that both are measured on the same probe volume. In order to achieve the desired accuracy of $\pm 2 \mathrm{e}-4$ on da/a, good control of the energy-channel relation of the detector is required (frequent recalibration on a fluorescence line, and corrective terms for intensity and non-linearity).

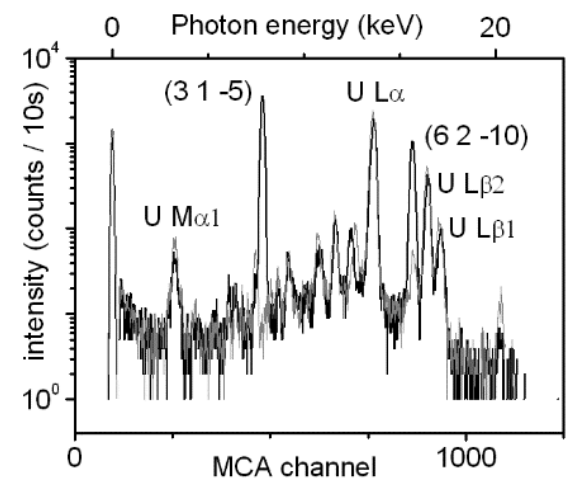

Fig. 5.3. Energy spectrum recorded after positioning the energy-resolved point detector to intercept the $(31-5)$ diffracted ray of a micron-sized $U_{2}$ grain. Such a spectrum is used in combination with the simultaneously collected Laue pattern to access the full strain tensor of the grain (see Robach et al., 2011). 


\subsubsection{Laue-DIC (Petit et al., 2011)}

Accurately measuring the absolute position of the diffraction spots down to 0.1 pixel may often be difficult (e.g. for irregular spot shapes) and it becomes interesting instead to measure their displacements with respect to a reference position, using digital image correlation (DIC). This should allow a more precise measurement of the coordinates of the $\mathbf{a}, \mathbf{b}, \mathbf{c}$ vectors (the "strained orientation matrix") in a differential mode, with respect to a reference matrix. A proof of principle of this method was demonstrated for the intragrain variation of the stress in the thickness of a bent $\mathrm{Si}$ wafer. This method potentially allows for the measurement of differences of orientation and strain i) between different points of the same grain (similar to high angular resolution electron back-scatter diffraction), and ii) between two different mechanical states of a same "material point" (or analysis volume) in a grain. This last application is under development. Figure 5.4 shows an example of strain-induced spot displacements measured by DIC.

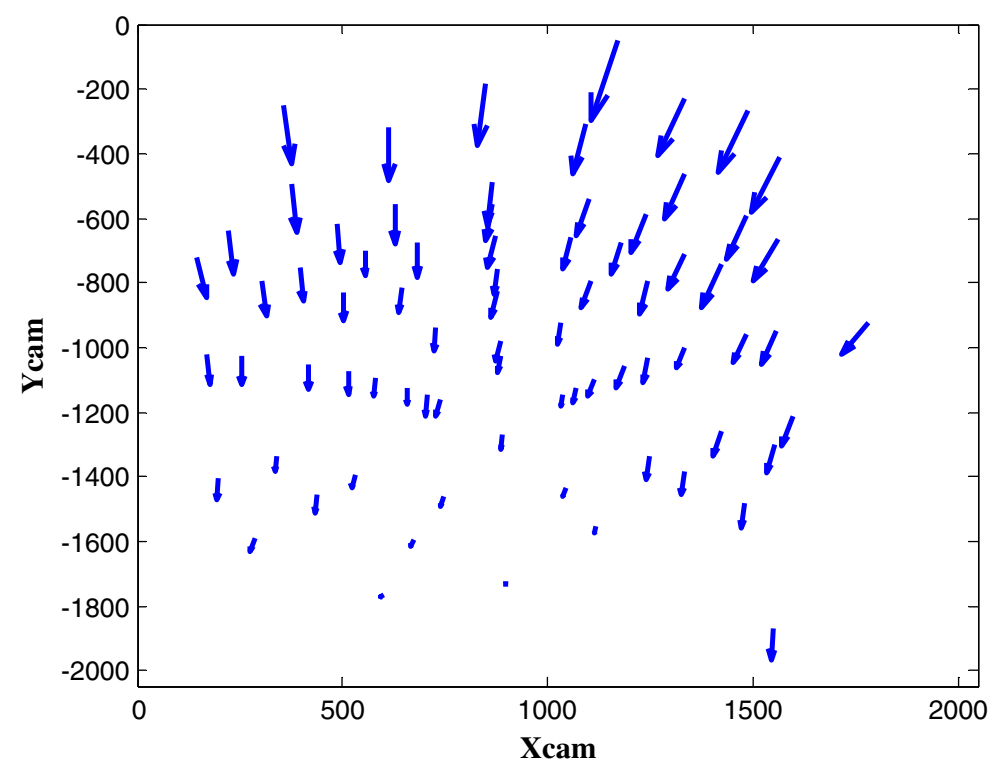

Fig. 5.4. Spot displacements $(\times 100)$, as determined by DIC, between two Si Laue patterns collected on the neutral fiber and on an end fiber of a bent Si wafer (thickness $1.82 \mathrm{~mm}$, tensile/compressive strain on the end fibers $\varepsilon_{y y}= \pm 7.5 .10^{-4}$, probed volumes $0.9 \mathrm{~mm}$ apart) (from Petit et al., 2011). 


\subsubsection{Instrument: perspectives}

\subsubsection{Setup}

The installation of an improved version of the microdiffraction setup is planned for 2012. The present supporting frame for the sample stage and $\mathrm{K}-\mathrm{B}$ mirrors will be replaced by an active vibration isolation table. New ellipsoidal K-B mirrors with fixed curvature, mounted on hexapodes, should provide both an improved beamsize $\left(200 \times 400 \mathrm{~nm}^{2}\right)$ and a photon flux density 16 times higher (thanks to larger acceptance and lower slope errors of the $\mathrm{K}-\mathrm{B}$ mirrors). Mapping during in situ mechanical tests on micro-objects requires fixed sample, therefore tests for mapping the samples by scanning the beam will be conducted. Longer-term projects involve adding a motorized stage for the $2 \mathrm{D}$ detector, in order to achieve better reproducibility of the experimental geometry (e.g. for the LaueDIC method). Also, an optical DIC setup is planned for monitoring the displacement field at the surface during mechanical tests with high plastic strain. In the initial state, the micro-Laue map is performed with measurement points placed on a regular 2D grid. In the deformed state, the micro-Laue map will be performed with a distorted grid based on the optical DIC measurements, which provide the displacement of each point of the initial grid. This is needed when the sample is subjected to a heavy deformation that produces a strongly inhomogeneous displacement field. The setting up of the single-grain online analysis of the Laue patterns should allow automatic 2D mapping of the hydrostatic strain using the white-beam method. The definition of procedures for routine use of the wire-scanning method is under way. Methods for reducing the beam "tails" in order to access weak signals in the tails of the spots will also be addressed.

\subsubsection{Software}

Improvements to the speed of analysis are planned. Peak search speed is already acceptable (5-10 seconds for 500 spots, depending on the method). Indexation speed is currently 2-3 minutes per image for six grains starting from 400 spots. Implementing changes of strategy (image matching, recognition of already indexed grains and recognition of twins) during indexation when treating a series of multigrain images should allow 
the acceleration of the process and increase the fraction of indexed spots. The module for displaying map results is under development.

\subsection{Examples of Application}

\subsubsection{Introduction}

Below are a few scientific questions that the users of the Laue microdiffraction setup have been trying to answer:

- What is the influence of fission products (e.g. dissolved He) in the mechanical stability of nuclear fuels? Do fission products change the balance of stress between grains of different orientations? And between the inner and the outer part of a grain? What is the epitaxial relation between a thick oxide and the unoxidized material below (Richard, 2012; Richard et al., 2012)?

- What is the characteristic distance of influence of a grain boundary in crystal plasticity processes (Devincre et al., 2008; Daveau, 2012)?

- Can Laue microdiffraction signals be predicted from model dislocation assemblies resulting from dislocation dynamics simulations (Gatti and Devincre, 2013)?

- What is the amount of initial residual stresses in devices such as solid oxide fuel cells obtained by assembling polycrystalline layers of different compositions? How are the stresses distributed between grains of different orientations? How do the stresses evolve when the device operates at high temperature and its components react with the incoming gases (Villanova et al., 2010, 2011)?

- What is the amount of the residual stress in 3D polycrystalline copper structures embedded in silicon used in microelectronics? How does this stress evolve when strong electrical currents flow in the conductor? Do certain grain orientations or multigrain arrangements favor electromigration and loss of electrical conduction? What would be the best microstructure in terms of reliability (Bleuet et al., 2013)?

- How do the changes of phase happen in shape memory alloys under mechanical load (Berveiller et al., 2011)? 
- How does the size of a crystal influence its plastic properties (Kirchlechner et al., 2011a, 2011b, 2011c, 2012)?

Three examples will be described: two briefly in Section 5.3.2, and a third in more detail in Section 5.4.

\subsubsection{Examples}

\subsubsection{Orientation in shape memory alloys during tensile tests}

Malard et al. (Berveiller et al., 2011) monitored the evolution of the field of the austenite and martensite phases in a millimeter-sized grain of a CuAlBe shape memory alloy during in situ tensile testing. The reversible conversion of the austenite phase into martensite phase under tensile loading confers super-elastic properties to the alloy, which allows it to recover its initial shape after unloading, even after $2.5 \%$ strain.

The authors monitored the changes in the orientation of the austenite unit cell in the vicinity of the newly formed martensite slabs, as a function of the applied macroscopic load. A global rotation of the austenite was observed on loading, of $2.5^{\circ}$ between $\varepsilon=5.10^{-4}$ and $\varepsilon=2.4 \%$ ( $\varepsilon=$ tensile strain). It went down to $1.3^{\circ}$ at $\varepsilon=1.4 \%$ then 0 at $\varepsilon=0$ on unloading. A reduction of the mosaicity of the austenite was observed on loading (from $1.2^{\circ}$ to $0.5^{\circ}$ between 0 and $2.4 \%$ ), and a corresponding increase on unloading $\left(1.1^{\circ}\right.$ at $\left.\varepsilon=0\right)$. The orientation map at $\varepsilon=1.4 \%$ on unloading also showed a rotation difference of $0.7^{\circ}$ between two austenite slabs separated by a martensite slab. This illustrates the decomposition of the original austenite grain into subdomains during loading, which merge again as a single domain on complete unloading (the mosaicity here is defined as inside a subdomain). Complementary 3D X-ray diffraction (3DXRD) experiments allowed the rotations of several grains to be measured, and revealed a dependence of the global rotation upon crystallographic orientation. The intragrain data from this study would probably benefit from an analysis by the Laue-DIC method, to gain insight into local stresses. Figure 5.5 shows the orientation map in the austenite domain at $1.3 \%$ tensile strain. 


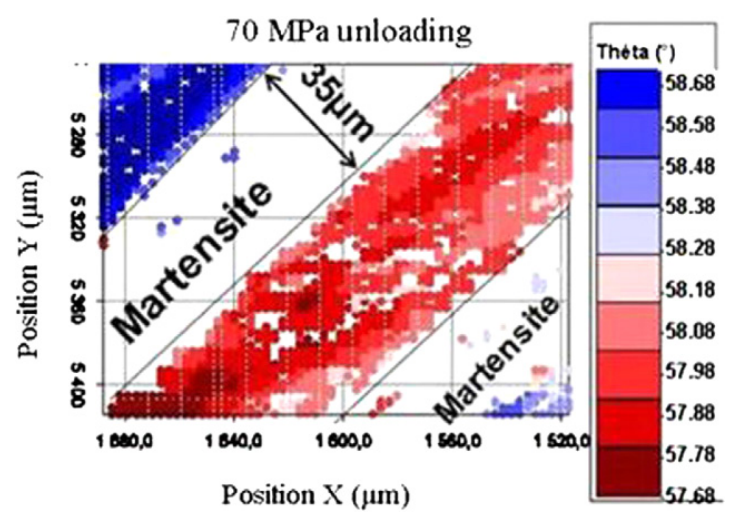

Fig. 5.5. Orientation map in the austenite domains of a CuAlBe shape memory alloy with a $1.3 \%$ tensile strain (unloading from a $2.4 \%$ strain). White regions correspond to slabs that changed from austenite to martensite structure during the straining process. Top left slab: $58.4<\theta<58.7$. Middle slab: $57.7<\theta<58.2$ (Berveiller et al., 2010).

\subsubsection{Stress in solid oxide fuelcells}

Villanova et al. $(2010,2011)$ measured the full stress tensors in several grains of the polycrystalline electrolyte layer $\left(\mathrm{ZrO}_{2}+\mathrm{Y}_{2} \mathrm{O}_{3}(8 \%), 7 \mu \mathrm{m}\right.$ thick) of a "half" fuel cell, for different "simulated" stages of the fuel-cell operation.

Solid oxide fuel cells (SOFCs) consist of a stack of several polycrystalline oxide layers. Local stresses originate from several phenomena:

- The difference of thermal expansion coefficient between the different materials, which changes the internal stress between room temperature and the operation temperature $\left(750-900^{\circ} \mathrm{C}\right)$.

- The strong anisotropy of the elastic rigidity tensor, which causes variations of the elastic response from one grain to the next for a given applied stress.

- The operation of the fuel cell, which changes the chemical composition of the interfacial layer located between the anode and the electrolyte. In this layer, which is initially part of the anode layer, the Ni may switch from an initial state of complete oxidation, to a fully reduced state and then to a fully re-oxidized state. The desired operating state is the fully reduced state, but a weak excess of oxygen with respect to hydrogen in the input gas, is sufficient to rapidly re-oxidize the interfacial layer. 


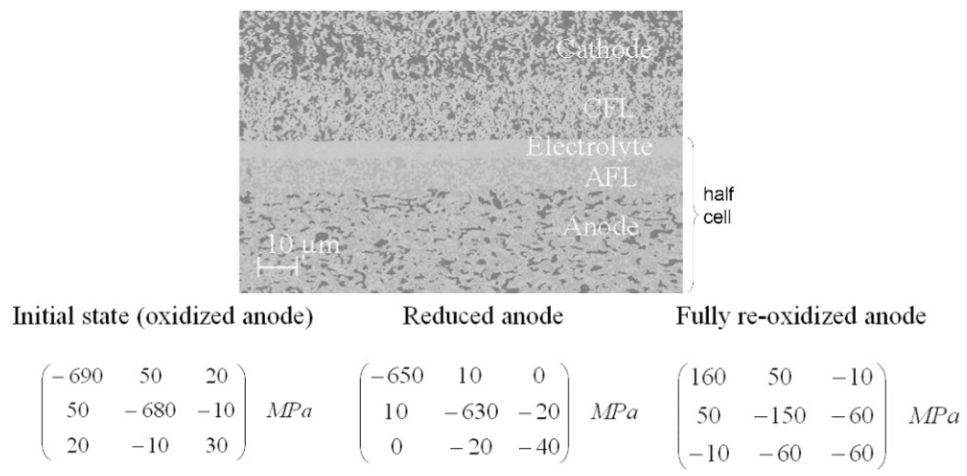

Fig. 5.6. Top: secondary electron microscope (SEM) image of an SOFC in cross section with the five layers of cathode, cathode functional layer, electrolyte, anode functional layer and anode. Bottom: variation of the electrolyte stress tensor in a half-cell, as averaged over about ten grains, from the combination of white-beam and monochromatic-beam Laue microdiffraction measurements, as a function of the cell state. These values are consistent with macroscopic measurements by the $\sin ^{2}$ (psi) method (see Villanova et al., 2011).

Here the fuel-cell operation and the associated thermal events were simulated by anneal under reducing $\left(\mathrm{H}_{2}\right)$ or oxidizing (air) conditions. This changed the oxidation state in the whole anode layer (and not only in the interface layer).

Figure 5.6 shows the variation of the electrolyte stress tensor as averaged over about ten grains, as a function of the cell state. During operation, a relaxation of the initial biaxial compressive stresses was observed, which was most significant during re-oxidation. Visual inspection of the cell under the microscope revealed cracks affecting the whole thickness of the electrolyte. Grain-to-grain measurements in the initial (highly stressed) state allowed the comparison of the stress and strain states between different orientations. Very high strains of the order of $2.10^{-3}$ were observed on the diagonal components. Deviations on the diagonal components up to $7.10^{-4}$ for the strain (mean $-2.3 .10^{-3}$ ) and $170 \mathrm{MPa}$ for the stress (mean $-680 \mathrm{Mpa}$ ) were observed with respect to the mean biaxial values in the layer plane. The simple descriptions (Voigt or Reuss) of the elastic behavior of the equivalent homogeneous medium are therefore mostly invalid when interpreting macroscopic measurements. A very weak stress was measured on the $\sigma_{z z}$ component for most of the grains $(-10$ to $+80 \mathrm{MPa}$ ), which was consistent with the location of the electrolyte layer at the surface of the half-cell. 


\subsection{In Situ Plastic Deformation of Single-Crystalline Micropillars}

\subsubsection{Introduction: plasticity in micropillars}

This study followed that of Uchic et al. (2004), whose authors showed a strong size effect in compressive tests in micro-objects: small singlecrystalline samples show a significantly larger flow stress than their macroscopic counterpart. In order to better understand the plasticity mechanisms that operate during mechanical tests on small objects, (Kirchlechner et al., 2011a, 2011b, 2011c, 2012) used Laue microdiffraction to monitor the fields of crystal orientation and orientation gradient in single-crystalline copper micropillars, during compressive and tensile tests, following the approach of Maaß et al. $(2006,2007)$.

Face-centered cubic (FCC) crystal plasticity is mediated by dislocation glide, at least for the temperature of interest here (room temperature). Dislocations are linear crystal defects which, by shear stress, can be heterogeneously nucleated at other crystal defects (e.g. at surface steps) or homogeneously multiplied from dislocation sources such as a FrankRead Source (Frank and Read, 1950; Hirth and Lothe, 1968).

Surface dislocation sources need a strong stress concentration to operate and are usually neglected, except for crystals that would contain no dislocation source in the volume.

Volume sources are typically an existing dislocation line pinned at one (single-arm source) (Hull and Bacon, 2001) or two anchor points (FrankRead Source). For a pillar with a small section, double anchoring of a dislocation line, in the glide plane of a given slip system, may have a small probability (if the starting dislocation density is small). The single-arm source may then be more probable than the double-arm source. The critical shear stress for this source is expected to be lower, as one end of the single arm progresses by escaping at the surface.

To be detectable by local diffraction, dislocations need to be immobile (i.e. stored) as mobile dislocations move too fast (approximately $1 \mathrm{~m} / \mathrm{s}$ ). A dislocation becomes temporarily or permanently stored by interacting with other fixed crystal defects (including point defects, dislocations, interfaces, etc.) (Hirth and Lothe, 1968).

Dislocation storage may be absent even when plasticity occurs. In a small object in particular, dislocations can escape at the surface, to 
which they are strongly attracted due to the image force. ${ }^{1}$ According to some authors - in a sufficiently small sample - all mobile dislocations are leaving the crystal, which leads to a hardening phenomenon called "dislocation starvation" (Greer and Nix, 2006).

The questions are then: do the acting dislocations of the plastic deformation stay long enough in the crystal to be observed? What is their density (how many dislocations per unit area)? Will dislocations playing more minor roles also be visible?

Stored dislocations may play at least three roles in the plasticity mechanism. Pairs will increase the lattice friction (through the forest effect) of the crystal with respect to the motion of dislocations on other slip systems. Unpaired dislocations may play very different roles: a pileup against a barrier, a structure typical of dynamic situations, will play a role of elastic energy storage and of stress concentration at the head of the pile. A wall, on the other hand, will provide, in static situations, the geometrically necessary lattice rotation for maintaining a shape obtained by plastic bending, with comparatively little stored elastic energy.

To understand the mechanism, not only the total number of dislocations (sorted by slip systems and edge/screw character) in the probe volume should be determined, but also the fractions playing the various roles.

Another question is therefore: if stored dislocations are detected, how can their role in the deformation mechanism be identified?

Section 5.4 is organized as follows. Section 5.4.2 gives the experimental details, Section 5.4.3 shows the results and Section 5.4.4 concludes. Further details are available in the appendixes. Appendix 5.A recalls the arguments that allow the extraction of dislocation densities from Laue microdiffraction measurements. Appendix 5.B tries to predict the local effects of the mechanical test on the diffraction.

\subsubsection{Experimental}

This study featured tensile and compressive tests on single-crystalline copper micropillars (3-7 $\mu$ min diameter) that were cut using a focused ion

${ }^{1}$ Surface escape is favored as it cancels the dislocation self-stress field. 
beam (FIB) workstation. The pillars were oriented in a single slip orientation to favor simple slip on system 1 . Special care was taken to avoid FIB damage at the surfaces.

The experimental challenge of this kind of test (in particular in terms of vibration) restricted the measurements with respect to the ideal possibilities of the technique:

- White beam was used but not monochromatic beam: geometrically necessary dislocations (GNDs) were probed but not statistically stored dislocations (SSDs).

- Laue patterns were analyzed in terms of orientations and spots shape/width, but not in terms of stresses (the appropriate accuracy, under $10 \mathrm{MPa}$, is not achievable with the standard analysis).

- Laue microdiffraction maps were collected in the unloaded state, interrupting the straining process: during the mechanical test, the beam probed a single point at the center of the sample. Indeed, the accelerations on the sample holder have destructive effects due to the difference in mechanical response of the two parts in contact.

\subsubsection{Results}

\subsubsection{Compressive tests: in situ tests on $7 \times 7 \times 21 \mu m^{3}$ pillars}

5.4.3.1.1. Experimental geometry as derived from the Laue pattern

The detailed description of the geometry is limited to one of the three pillars (noted "B") studied by Kirchlechner et al. (2011a). Figure 5.7 shows a stereographic projection giving the crystallographic orientation of the various geometrical elements of the problem. The interest of this figure is detailed in Appendix 5.B.

Table 5.1 summarizes the geometrical results for the 12 slip systems. Only systems 1 and 2 kept the same rank (in terms of Schmid factor) throughout the experiment: in the mobile part of the sample, the other systems changed rank during the test. Unless otherwise specified, a system rank refers to the initial state.

Angle values that follow are given within $\pm 0.3^{\circ}$, as the axes of the sample frame (as defined from experimental geometry: incident beam impact point - detector) may slightly deviate from the sample and punch motor axes. 


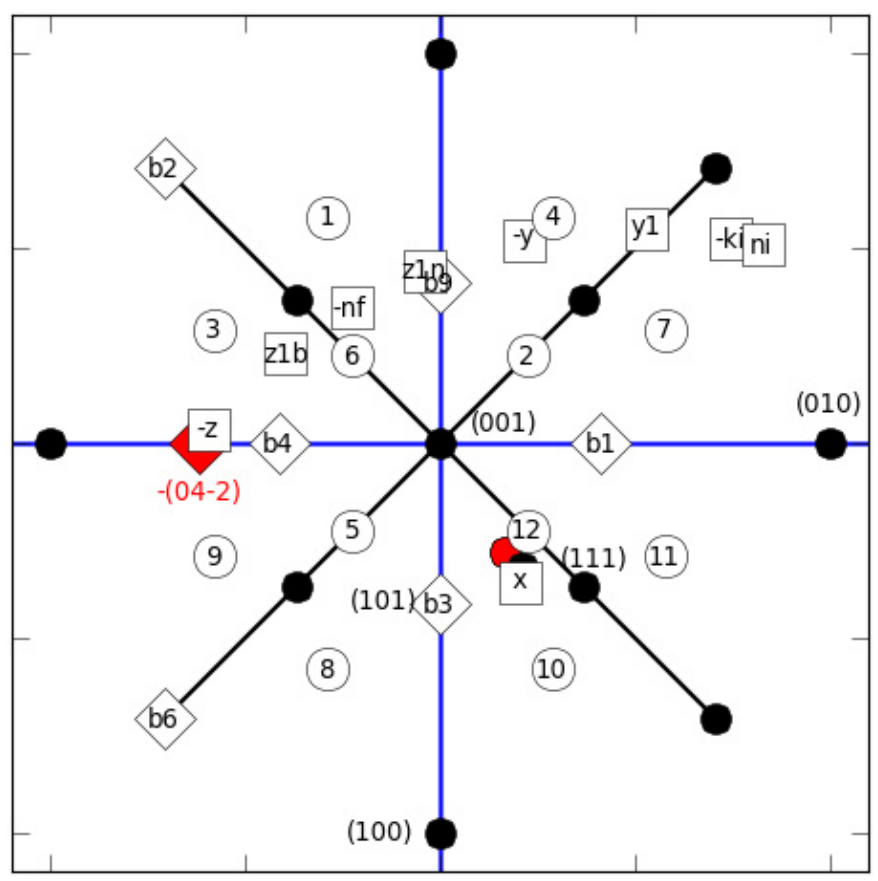

Fig. 5.7. Stereographic projection showing the geometry of the compression experiment for pillar B in reciprocal space (q-space), in the initial state. The compressive axis is along $\mathbf{x}$ (horizontal) (close to the $2 \theta$ axis and the $\mathrm{x}$ cam axis of the $2 \mathrm{D}$ detector). The horizontal incident beam $\mathbf{k}_{\mathbf{i}}$ is perpendicular to $\mathbf{x}$. The pillar lateral faces are close to horizontal (f face, normal $\mathbf{n}_{\mathbf{f}}$ ) and vertical (i face, normal $\mathbf{n}_{\mathbf{i}}$ ). $\mathbf{n}_{\mathbf{i}}$ is the outer normal to the entrance face of the pillar, $\mathbf{n}_{\mathbf{f}}$ the outer normal to the exit face, which faces the detector screen. See also Figs. 5.B1 and 5.B3 in Appendix 5.B. The numbered circles show the $\langle 112\rangle$ (= $\mathbf{L}_{-}$edge) rotation axes corresponding to the various slip systems. $\mathbf{b}_{\mathbf{1}}$ is the Burgers vector of system 1 (highest Schmid factor). The $\mathrm{P}_{1}$ planes are (1-11). $\mathbf{b}_{\mathbf{8}}=\mathbf{b}_{\mathbf{1}}$. The (04-2) reciprocal point is shown as a large diamond. After the $\mathbf{k}_{\mathbf{f}}=\mathbf{k}_{\mathbf{i}}+\mathbf{q}$ projection it gives a spot close to the center of the detector. The axes $\mathbf{y}_{\mathbf{1}}=\left(\mathbf{x} \times \mathbf{n}_{\mathbf{1}}\right) \times \mathbf{x}, \mathbf{z}_{\mathbf{1} \_\mathbf{b}}=\mathbf{x} \times \mathbf{b}_{\mathbf{1}}$ and $\mathbf{z}_{\mathbf{1} \_n}=\mathbf{x} \times \mathbf{n}_{\mathbf{1}}$ associated to the frustrated lateral displacement and bending/rotation of the pillar are shown. The systems with the best sensitivity for detecting GNDs (b close to $\left.\mathbf{k}_{\mathbf{i}}\right)$ are 6 and $12\left(\mathbf{b}_{\mathbf{6}}=\mathbf{b}_{\mathbf{1 2}}\right)$.

The compressive axis $\mathbf{x}$ is initially along the (hkl) $=[52.895 .79]$ direction at $8.8^{\circ}$ from the [538] direction which maximizes the difference of Schmid factor between system 1 and system 2, and at $3.8^{\circ}$ from the targeted [324] direction. The Burgers vector, $\mathbf{b}_{\mathbf{1}}$, and the normal, $\mathbf{n}_{\mathbf{1}}$, to the $\mathrm{P}_{1}$ planes are respectively at an angle of $41.4^{\circ}$ and $33.9^{\circ}$ with respect to $\mathbf{x}$. $\mathbf{b}_{1}$ deviates from the line of greatest slope (along $\mathbf{x}$ ) of the $\mathbf{P}_{\mathbf{1}}$ plane: the $\left(\mathbf{x}, \mathbf{b}_{1}\right)$ plane makes an angle of $40.2^{\circ}$ with the $\left(\mathbf{x}, \mathbf{n}_{1}\right)$ plane. 
Table 5.1. Summary of the geometrical results for the 12 slip systems: Schmid factor, $\langle 112\rangle$ rotation axis, expected elongation for the $(04-2)$ Laue spot (without the L_b term), amplitude ( $\sin \alpha$ in q-space, $\mathrm{dxy}$ in detector space), and direction (tilt $=(-$ dycam/dxcam) (degrees) in detector space). The dxy in pixels is calculated for $\mathbf{d q} / \mathbf{q}=0.01 \mathrm{rad}$. L_b is the length of the diffracting volume along $\mathbf{b}$ in microns using $1 \times 1 \times 7 \mu \mathrm{m}^{3}$ diffracting volume. System rank and Schmid factor are also given for $\varepsilon=0.2$ (see Appendix 5.A for further details).

\begin{tabular}{lcccccccc}
\hline $\begin{array}{l}\text { rank } \\
\varepsilon=0\end{array}$ & $\begin{array}{c}\text { Schmid } \\
\text { factor }\end{array}$ & $\begin{array}{c}\text { dxy } \\
\text { pixels }\end{array}$ & $\begin{array}{c}\text { tilt } \\
\text { degrees }\end{array}$ & $\begin{array}{c}\text { L_b } \\
\mu \mathrm{m}\end{array}$ & $\begin{array}{c}\text { sin } \\
\alpha\end{array}$ & $\begin{array}{c}\text { rotation } \\
\text { axis }\end{array}$ & $\begin{array}{c}\text { rank } \\
\varepsilon=0.2\end{array}$ & $\begin{array}{c}\text { Schmid } \\
\text { factor }\end{array}$ \\
\hline 1 & 0.419 & 13 & 33.9 & 4.6 & 0.84 & $21-1$ & 1 & 0.443 \\
2 & 0.38 & 13.3 & -38.3 & 1.4 & 1 & -112 & 2 & 0.395 \\
3 & 0.243 & 14.1 & 48 & 2.6 & 0.41 & $12-1$ & 5 & 0.216 \\
4 & 0.242 & 11.9 & 5.2 & 3.6 & 0.98 & -211 & 3 & 0.3 \\
5 & 0.177 & 17.3 & -89.3 & 1.4 & 0.68 & $-11-2$ & 6 & 0.144 \\
6 & 0.176 & 12.4 & -22.5 & 6.9 & 0.68 & $-1-12$ & 4 & 0.227 \\
7 & 0.138 & 12.9 & -32.2 & 2.6 & 0.84 & $1-2-1$ & 7 & 0.095 \\
8 & 0.111 & 15.3 & 60.6 & 4.6 & 0.84 & $2-11$ & 10 & 0.071 \\
9 & 0.066 & 14.1 & 48 & 3.3 & 0.41 & $-12-1$ & 8 & 0.073 \\
10 & 0.065 & 16.8 & 77.2 & 3.6 & 0.98 & 211 & 9 & 0.072 \\
11 & 0.038 & 17.2 & -84.2 & 3.3 & 0.84 & 121 & 11 & 0.048 \\
12 & 0.027 & 17.1 & -80.6 & 6.9 & 1 & 112 & 12 & 0.024 \\
\hline
\end{tabular}

By mounting, the pillar's i face is not exactly perpendicular to the $\mathrm{X}$-ray beam (see Fig. 5.B3): the outer normal to the entrance face points downwards by $5^{\circ}$ (manual rotation). $\mathbf{L}_{\mathbf{1} \_ \text {edge }}$ makes an angle of $8.5^{\circ}$ with the glide traces " 1 " on the i face $\left(90.5 \%\right.$ edge), and of $76.5^{\circ}$ with the glide traces " 1 " on the f face ( $85 \%$ screw). Mixed " 1 " dislocations possibly piled up against the i faces therefore have a strong edge character. The conditions are favorable for detecting them with the beam at the pillar's center (along nf) (see Appendix 5.B).

\subsection{Mechanics results: stress-strain curves}

for the three pillars

Figure 5.8a shows the stress-strain curves for the three pillars. An unloading phase was inserted at $\varepsilon=0.1$. This allowed the mapping of the sample, and the checking of the alignment of the sample's top surface with respect to the flat punch counter body. Maps were also performed at $\varepsilon=0$ and $\varepsilon=0.2$ in the unloaded state. 


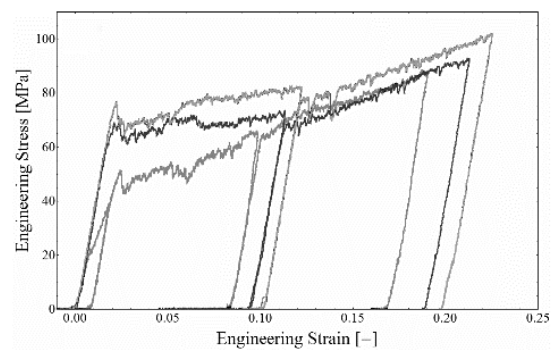

(a)

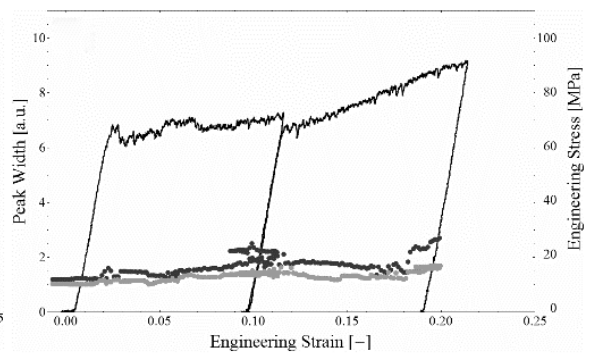

(b)

Fig. 5.8. Stress-strain curves for the compression of three $\left(7 \times 7 \times 21 \mu \mathrm{m}^{3}\right)$ copper single-crystalline pillars cut for single-slip. Pillars: A (dark gray), B (black) and C (light gray, topmost curve). Temporary unloading around $\varepsilon=0.1$ allowed the collection of Laue maps. An additional unloading step was performed around $\varepsilon=0.02$ for pillar A. (b) Full width at half maximum (FWHM) of the $(04-2)$ spot superimposed with the stress-strain curve for pillar B. Two FWHM values are given to characterize the spot shape on the 2D detector. Dark gray: along the direction of elongation; light gray: perpendicular to the direction of elongation (see Kirchlechner et al., 2011a).

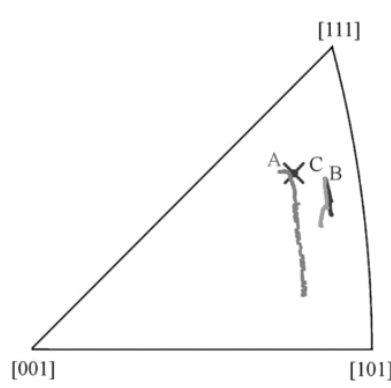

(a)

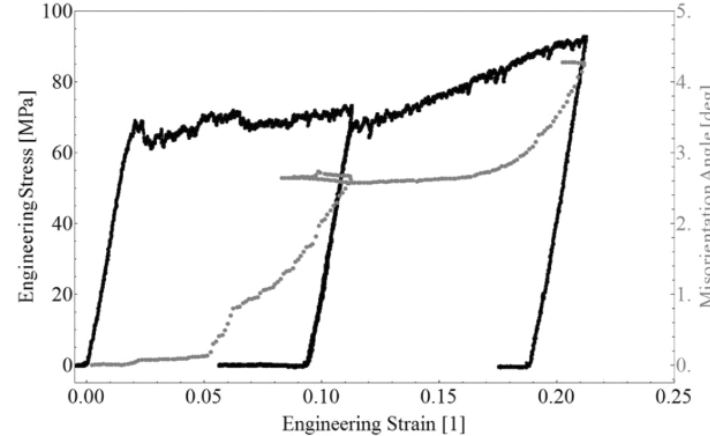

(b)

Fig. 5.9. (a) Inverse pole figure showing the trajectory of the load axis $\mathbf{x}$ in the stereographic projection of the crystal during compression for the three pillars (see Kirchlechner et al., 2011a). (b) Stress-strain curve for pillar B (black) and rotation angle of the crystal unit cell (gray).

\subsection{Diffraction results}

\subsection{Lattice orientation at the center of the pillar versus strain}

Figure 5.9a shows the displacement of the compressive axis $\mathrm{x}$ (fixed) in the stereographic triangle (mobile) of the crystal during the compression, for the three pillars. 
The experimental rotation axis between the base and the center of pillar $\mathrm{B}$ at $\varepsilon=0.2$ was close to the $\mathbf{z}_{1 \_\mathbf{n}}$ axis (within $11^{\circ}$, see Fig. 5.B1). Rotation around $\mathbf{z}_{1 \_\mathbf{n}}$ axis is expected for slip parallel to the $\mathrm{P}_{1}$ plane, when the whole pillar undergoes homogeneous shear up to the top surface (see Appendix 5.B). The experimental axis is therefore consistent with slip parallel to the $\mathrm{P}_{1}$ plane.

Figure 5.9b shows the experimental evolution vs the applied strain of the rotation angle of the crystal. Up to $\varepsilon=0.05$, only a very small rotation was observed. Significant rotation then suddenly started. On unloading at $\varepsilon=0.1$, the angle remained almost stable (variation of $0.27^{\circ}$ ), which showed that the system was well aligned. On reloading after $\varepsilon=0.1$, only very small rotation was again observed until $\varepsilon=0.18$.

The angle vs strain curve, combined with the SEM image (Fig. 5.10), suggests two regimes in the $\varepsilon<0.1$ strain range:

(1) A first "unguided" regime, with slip without rotation, for $\varepsilon<0.05$. The absence of rotation suggests a slicing of the pillar along $\mathrm{P}_{1}$ planes, into a sheared middle slice and two fixed end parts. This suggests a lateral motion of the top end, allowed by some lack of lateral stiffness (angular play) in the guiding of the punch. The test would therefore be more ideal than expected for perfectly guided non-rotating ends.

(2) A second regime of compression for $\varepsilon>0.05$, with a rotation around $\mathbf{z}_{1}$ n, without lateral movement of the counter body with respect to the sample base. This "guided x motion" regime would start when the gap between the guiding rail and the punch-holder closes or when a sufficient high lateral force arises. Assuming slip along $\mathbf{b}_{\mathbf{1}}$, the calculation with $\varepsilon=0.05$ and $\mathrm{L} 0=21 \mu \mathrm{m}$ leads to an estimated initial lateral movement of $1 \mu \mathrm{m}$.

A question is then the mechanical reason for the reorientation of the interface between the fixed and sheared parts of the pillar at $\varepsilon=0.05$. Here this seems to be the locking of the yz translation, the fixed orientation of the top surface would come into play only because of this locking.

In Fig. 5.9b, the change of slope on re-loading after $\varepsilon=0.1$ could then be interpreted as a "reset to zero" of the boundary conditions, with the punch relaxing back to its center position (laterally), and the laterally displaced top surface of the pillar contacting a new area on the punch surface. The 


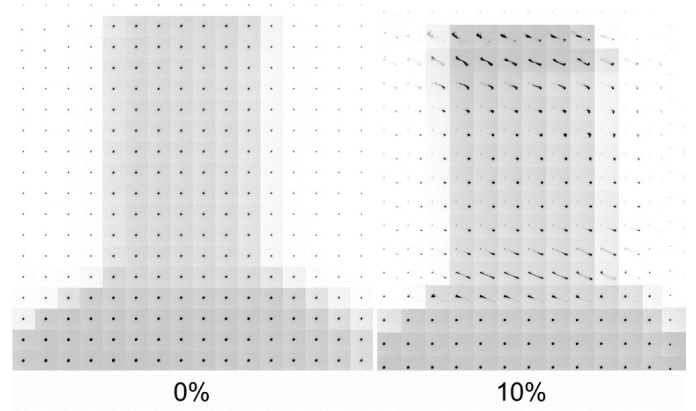

(a)

(b)

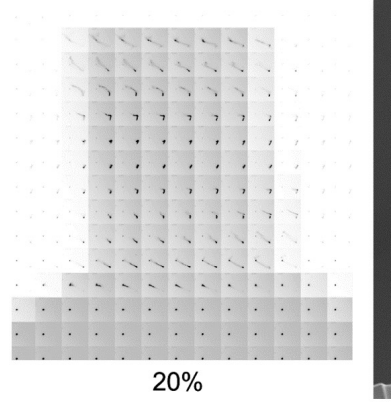

(c)

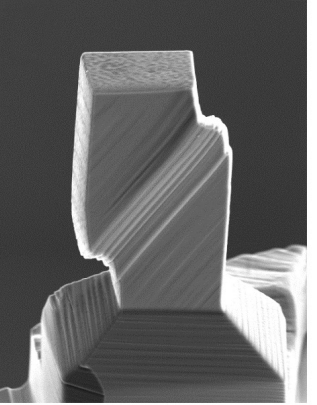

(d)

Fig. 5.10. (a-c) Laue mosaic maps of the $\left(\begin{array}{lll}0 & 4 & 0\end{array}\right)$ spot in the unloaded state at different strains for pillar B: 0 (a), 0.1 (b) and 0.2 (c) (box size $275 \times 255$ pixels). Each box is centered on the same fixed pixel position on the detector. (The signs of the $\mathrm{x}$ and $\mathrm{y}$ pixel axes are reverted with respect to Fig. 5.11). (d) An SEM image of pillar B after the test. The most visible lateral face of the pillar is the $\mathrm{f}$ face, parallel to the X-ray beam in the micro-Laue experiment.

unguided mode would restart (this time with a slight angular offset between the load axis and the mean pillar axis) and pursue until approximately 0.18 strain. A lack of angular stiffness of the punch therefore seems more likely than a slip of the top surface with respect to the punch, as an explanation for the unguided regime. This suggests that interrupting the test more frequently would make it more ideal.

\subsection{Spot shape and width}

Spot width vs. strain for the three pillars Figure 5.8b shows the stressstrain curve during the compression of pillar B and, superimposed, the 
evolution of the FWHM of the (04-2) spot. The corresponding curves for pillars A and C can be found in the reference. During the test, the X-ray beam was at the center (axially and laterally) of the pillar.

Searching for direct correlations between the stress-strain curve and the FWHM-strain curve proves dangerous, as the first measurement is global while the second is local. For example, comparing pillars B and C showed that the delay between the end of the linear stress regime and the start of the FWHM increase changed between the two pillars. For pillar B these two events coincided, while for pillar C the FWHM increase occurred 0.015 later in strain.

The spot FWHM appeared more sensitive than the stress to the details of the test. Comparing pillars B and C showed that, after the switch to the guided mode around $\varepsilon=0.05$, enormous differences on the FWHM appeared between the two pillars, while the stress difference was only of $10 \mathrm{MPa}$. For pillar $\mathrm{C}$ the spot width increased by a factor of seven over a strain range $\Delta \varepsilon$ of 0.02 . For pillar B it increased only by a factor two over a 2.5 times larger strain range $(\Delta \varepsilon=0.05)$. The "multi-spot" width for pillar $\mathrm{C}$ at $\varepsilon=0.2$ was about five times larger than for pillar $\mathrm{B}$.

The data on pillar A showed that a reversible part in the GND storage could exist, most likely due to an unpiling of piled up dislocations. When loading this pillar to $\varepsilon=0.02$, the spot FWHM increased from 1.5 to 9 , and when unloading it to $\varepsilon=0$, the FWHM decreased back to 5. This particular behavior occurred because this pillar was initially stressed in bending due to a misalignment. This raises questions about a possible unstoring of GNDs between the test (under load) and the measurement of Laue maps (without load), in particular in case of dynamic storage of GNDs against the pillar faces.

Laue maps of pillar $B$ for three strain states (after unloading) and postmortem SEM Figure 5.10a-c show the mosaics of the (0 40$)$ spot at three stages of the stress-strain curve: initial state, after $\varepsilon=0.1$ and after $\varepsilon=0.2$. Figure 10d shows the SEM image of the pillar after the test.

In the Laue maps, a progressive reduction of the "ideal" central region (where the spot is sharp and round) was observed, and correspondingly an extension of the region influenced by the non-ideal boundary conditions (where the spot was elongated, with several elongation directions at 
$\varepsilon=0.2$ ). At $\varepsilon=0.1$, the lattice orientation was nearly constant at the center of the pillar, over about $60 \%$ of the height, evidencing a test close to ideal.

Analysis of the spot elongations for pillar B Figures 5.11a-d show the 2D intensity profile of the $(04-2)$ spot in the center of pillar B at different points of the stress-strain curve. Figure 5.11e shows a schematic of the Laue pattern. Figure 5.11f shows the theoretical elongations of the (04-2)

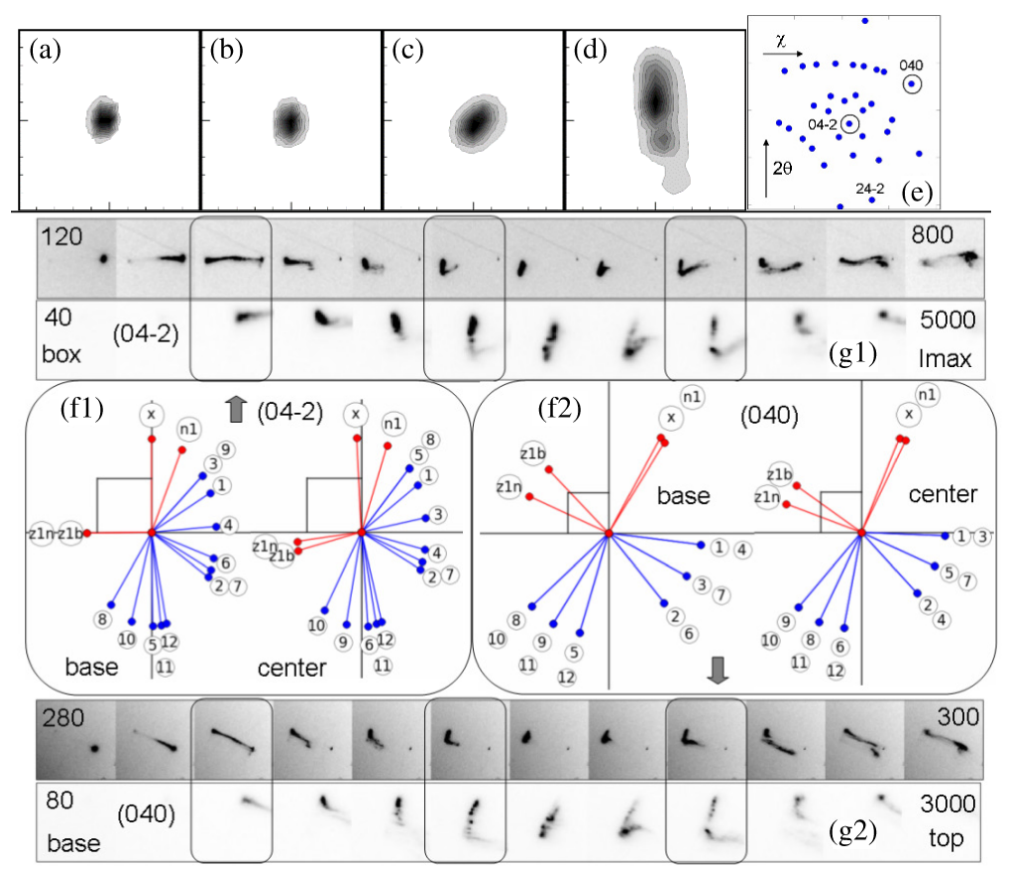

Fig. 5.11. (a-d) A 2D spot profile of the $(04-2)$ Laue spot under load at different strains at the center of pillar B (box size $20 \times 20$ pixels). Strain values: (a) 0 , (b) 0.02 just after the start of the plastic regime, before the load drop, (c) 0.11 just before the first unloading, (d) 0.22 just before the second unloading, (e) Laue pattern showing the $(04-2)$ and (040) spots. (f1, f2) theoretical elongation directions of the two spots for edge GNDs on the 12 slip systems, and for rotations around the $\mathbf{x}, \mathbf{z}_{1} \_\mathbf{n}, \mathbf{z}_{1} \_\mathbf{b}$ and $\mathbf{n}_{\mathbf{1}}$ axes. The square gives the 10-pixel scale. (g1, g2) Two-dimensional spot profiles of the two spots for several positions ( $\mathrm{dx}$ step $=2 \mu \mathrm{m})$ along the pillar axis at $\varepsilon=0.2$ after unloading. Two combinations of pixel box size and maximum intensity are given, to highlight the differences of elongation direction between the high-intensity region and the low intensity tails. The sharp "satellite" present in all the images is due to the $10 \mu \mathrm{m}$-radius low-intensity tails of the X-ray beam hitting the (much thicker) base of the pillar. 
and (040) spots for GNDs on the different slip systems and for rotations around the $\mathbf{x}, \mathbf{z}_{1 \_\mathbf{b}}, \mathbf{z}_{1 \_\mathbf{n}}$ and $\mathbf{n}_{\mathbf{1}}$ axes. All elongations correspond to a rotation of $0.01 \mathrm{rad}$ (i.e. without the $\mathrm{L}_{-} \mathrm{b}$ term). Elongations are given for two orientations of the crystal: in the base (= initial state) and in the center of the pillar at $\varepsilon=0.2$. Elongation directions may rotate by up to $15^{\circ}$ between the two situations. Figure $5.11 \mathrm{~g}$ shows the spot shapes at different positions along the pillar axis in the unloaded state at $\varepsilon=0.2$, at the center of the pillar (transverse-wise).

From Fig. 5.8b and Figs 5.11a-c, up to $\varepsilon=0.18$, the behavior at the center of pillar was close to ideal - only a small increase of the spot width was observed. Near the load drop at the end of the elastic regime, there was even a decrease of FWHM with increasing strain (Fig. 5.8b).

At $\varepsilon=0.1$, the minor elongation (Fig. 11c) approximately corresponds to a mix of systems 1 and 4 (ranked as 3 for $\varepsilon=0.1$ ), as checked from the elongation of other spots. The presence of system 4 may be due to plastic bending around the $\mathbf{z}_{1 \_\mathbf{n}}$ axis, this axis being close to $\mathbf{L}_{\mathbf{4} \_ \text {edge }}(\langle 112\rangle$ of system 4, see Fig. 5.7). Around $\varepsilon=0.18$ the spot split (Figs 5.8b and 5.11d), with an elongation corresponding to a rotation around the $\mathbf{n}_{\mathbf{1}}$ axis (see Figs 5.11g1 and 5.11g2) (perpendicular to P1) (as checked on other spots). This twisting of the lattice around $\mathbf{n}_{\mathbf{1}}$ could indicate torsion acting with an axis close to $\mathbf{n}_{\mathbf{1}}$. Estimating the direction of the corresponding gradient is necessary to go further in the interpretation. From Figs 5.11 and 5.10 the gradient direction has a large component along $\mathrm{x}$ and almost no component along $\mathbf{n}_{\mathbf{f}}$. The component along $\mathbf{n}_{\mathbf{i}}$ cannot be extracted here.

The other pillars (A and $\mathrm{C}$ ) also feature split spots at $\varepsilon=0.2$, with an elongation close to vertical for the central spot. The rotation around $\mathbf{n}_{\mathbf{1}}$ may therefore be a general "high strain" feature.

In Fig. 5.11f, boxes 6 and 9 (left box $=1$ ) feature an intense split spot with an elongation direction similar to the one at the center of the pillar (rotation axis $\mathbf{n}_{\mathbf{1}}$ ), and a low intensity streak close to the expected elongation for system 1 . The comparison with the SEM image suggests that here the X-ray beam crosses the glide traces on the $\mathrm{i}$ face. The diffuse streak could be due to " 1 " dislocations stored against the i face. This would indicate a resistance of the $i$ face to the escape of edge dislocations. 


\subsubsection{Tensile tests: ex situ study of $3 \times 3 \times 15 \mu \mathrm{m}^{3}$ pillars previously strained in situ in the SEM}

\subsection{Stress-strain curves for the three pillars and SEM of a high-strain pillar}

Three pillars were strained in the SEM (Kirchlechner et al., 2011b), two up to $0.25\left(\mathrm{P}_{2}\right.$ and $\left.\mathrm{P}_{3}\right)$ and the third to $0.04\left(\mathrm{P}_{1}\right)$. They were then studied post mortem using Laue microdiffraction.

Figure 5.12 shows the SEM image of pillar $\mathrm{P}_{2}$ after loading to $\varepsilon=0.25$ and unloading, and the stress-strain curves of the three pillars. For pillars $\mathrm{P}_{2}$ and $\mathrm{P}_{3}$, the SEM movie allowed the linking of the minor load drops of the stress-strain curves to the appearance of glide traces on the surface. The major load drop (at $6 \%$ for $\mathrm{P}_{2}$ and $3 \%$ for $\mathrm{P}_{3}$ ) was linked to a "geometrical softening" associated to the appearance of a very high surface step. This provided an example of an uneven distribution of slip events along the pillar axis that localized the bending momentum imposed by the boundary conditions near the slip band (see Appendix 5.B).

\subsection{Micro-Laue line scan and SEM of the low-strain pillar}

Figure 5.13 shows the SEM image of pillar $\mathrm{P}_{1}$ taken after loading to $\varepsilon=$ 0.04 and unloading, and the mosaic of a Laue spot for a line scan along the pillar. As in the other pillars, the slip along the $\mathrm{P}_{1}$ plane concentrated in a rather short length in the middle of the pillar, which led to a bending of the pillar in the middle. The bending was apparently partly plastic and partly elastic - on unloading the relaxation of the elastic part induced a rotation of the "head part" with respect to the "base part" of the pillar.

At each position on the sample, the Laue spot showed a satellite due to the signal from the rest of the sample (e.g. the thick parts, head and base), which was created by the $10 \mu \mathrm{m}$-wide low-intensity tails of the X-ray beam. Along the pillar, the spot position switched between two positions corresponding to two orientations, the one for the base and the other for the head. In the middle, where the slip occurred, spot elongation was observed along the line connecting the two "end" positions. This line corresponded to the storage of GNDs on system 3, although the slip (as checked by SEM) was in the $\mathrm{P}_{1}$ plane. 

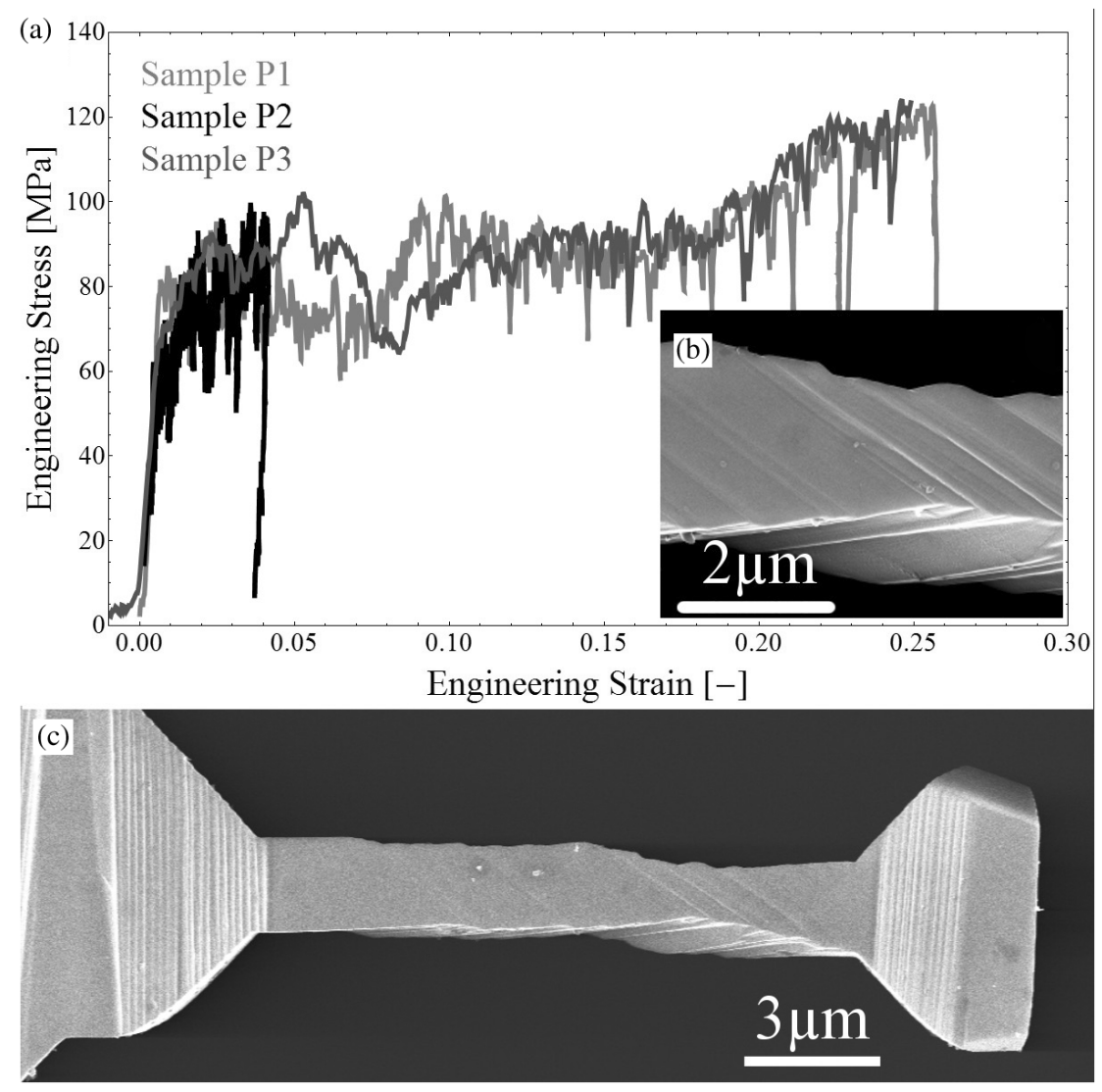

Fig. 5.12. (a) Stress-strain curves collected in the SEM during in situ tensile tests on samples $\mathrm{P}_{1}, \mathrm{P}_{2}, \mathrm{P}_{3}$, while collecting a SEM movie of the test. (b) Detail of the SEM image showing the high surface step. (c) SEM image of tensile sample $\mathrm{P}_{2}$ after 0.25 strain (unloaded). (see Kirchlechner et al., 2011b).

A tentative interpretation is the following. A geometric analysis, similar to the one carried out in Section 5.4.3.1.1, may lead to a $\mathbf{z}_{1 \_\mathbf{b}}$ (or $\mathbf{z}_{\mathbf{1} \_\mathbf{n}}$ ) bending axis close to $\mathbf{L}_{\mathbf{3} \_ \text {edge }}$ (the $\langle 112\rangle$ axis of edge dislocation lines of system 3). The bending associated to slip 1 would then have helped to activate system 3 .

\subsubsection{Conclusion and perspectives}

Laue microdiffraction allowed a refined exploration of the details of the mechanical test, and provided very rich information. It evidenced significant 


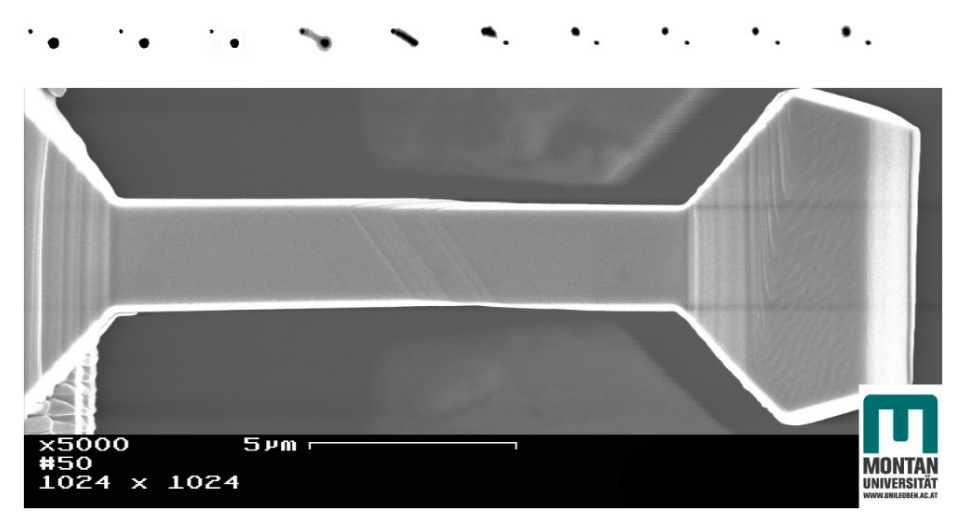

Fig. 5.13. Bottom: SEM image of tensile sample $\mathrm{P}_{1}$ after 0.04 strain (unloaded). Top: mosaic of the Laue spot for a line scan along the pillar.

differences between tests that would look similar from observation of the stress-strain curves.

The compression study provides a tentative ranking of the various types of measurements according to sensitivity to the test behavior. Stress-strain curves come first (not very sensitive), then spot width (comparison between pillars $\mathrm{B}$ and $\mathrm{C}$ ), then lattice rotation (comparison between angle and spot width for pillar B).

The evaluation of the role played by the lateral faces requires a particular strategy - for the compression of pillar B, the small height of the gliding region required a measurement outside of the pillar center (axis-wise) to detect the storage of edge GNDs on system 1 against two of the lateral faces.

The data described here contain an enormous amount of information, and the analysis could be pursued, in particular, to extract the stresses under load, using the Laue-DIC method for improved accuracy. The goal would be to quantify the degree of ideality of the test in the central region (see Appendix 5.B for the definition of an "ideal" test). The detailed analysis of the lattice rotation axis vs strain should also shed light on the operating bending torques.

For future studies, the combination with monochromatic-beam measurements would be desirable for monitoring all the dislocations, not only the GNDs. 
Recommendations on the cutting of pillars can be given, in order to simplify the non-ideal part of the test:

- Putting $\mathbf{b}_{1}$ parallel to one of the lateral faces would help - the SEM shape of the strained pillar would immediately provide the slip direction (glide traces on only one of the faces for slip 1). A possible dislocation pileup against the other faces would then be the most straightforward to interpret, with pure edge dislocations.

- Keeping the same crystalline orientation of the lateral faces for all the pillars would help the comparison of the results on the different pillars. With regards to the instruments, displacing the beam instead of the sample (using optical elements at a sufficient distance to avoid inducing vibrations on the sample) would be helpful:

- Mapping under load would help checking the good behavior of the test, and would provide dynamic maps of the stress and the GND densities. The degree of stress localization during the test could then be estimated, and the regions of stress concentration/cancellation (neutral fibers) could be located.

- The beam could be moved during the test, in order to always probe the same material point.

These studies highlight a well-known fact: that plasticity (i.e. shear) in small confined crystals tends to add bending and torsion stresses to initially uniaxial stresses. For low strains, if slip events are well distributed along the pillar (as for the compression of pillar B), these effects can be neglected up to strain values around 0.15 , and it is possible to observe the intrinsic behavior of the material at the center of the pillar.

In the mechanical tests on single crystals, the separation of the effects caused by the method (constraints causing torque and bending) and effects caused by the material (dislocation source statistics, formation of inner dislocation barriers) will be key to understanding the material properties and developing appropriate material models.

\section{Acknowledgements}

We are grateful to B. Devincre for his important contribution through numerous discussions. The contents benefited from stimulating discussions 
with, among others, H. Palancher, A. Richard (wire-scanning method), C. Le Bourlot, C. Maurice, L. Saintoyant (Laue-DIC method and EBSD), G. Daveau, T. Hoc (plasticity), S. Labat, S. Escoubas and O. Thomas (diffraction). The MECANO GdR provided the opportunities for many of these discussions. Furthermore, we want to thank C. Motz, W. Grosinger, P.J. Imrich, M. Kapp, J. Keckes and G. Dehm for assistance and support during in situ micromechanics.

\section{Appendix 5.A. Detection of Stored Dislocations Using Laue Microdiffraction}

\section{A.1. Introduction}

Appendixes A and B present the theoretical tools used in Section 5.4 to calculate spot elongation directions given some hypotheses on rotation axes/activated glide systems and to analyze spot shapes and displacements in Laue maps. Appendix 5.A discusses the passage from lattice rotations to diffraction, while Appendix 5.B discusses the passage from mechanics to lattice rotations.

In plastically deformed materials, the quantity that affects the diffraction is not plastic strain itself but its gradient. More precisely, it is the gradient of shear, and the associated dislocation storage. This causes spatial variations in the orientation of the crystal lattice, each dislocation acting as a small quantum of orientation gradient of the lattice.

Detailed mathematical description of the strain/orientation fields associated to dislocations (Hirth and Lothe, 1968; Hull and Bacon, 2001) and the associated changes in diffraction signals (Wilkens and Bargouth, 1968; Krivoglaz, 1995; Ice and Barabash, 2007; Kaganer and Sabelfeld, 2010; Gatti and Devincre, 2013) can be found in the cited references and in Chapter 1.

The discussion here is limited to the white-beam mode, unless otherwise specified. There are two methods with which to study orientation gradients. The first uses the shapes of Laue spots, and the second uses the spots' displacements between neighboring points on the sample.

\section{A.2. Orientation spread (GNDs): single-point measurements}

The search for orientation gradients can first be performed by maintaining the micro-probe volume at a fixed position with respect to the sample. 
The measured quantity is then the shape and characteristic length(s) of the diffraction spots on the 2D detector. Comparing the elongation directions of the different spots allows the identification of a possible single-rotation axis.

For the FCC lattice, the goal is to detect $\langle 112\rangle$ rotation axes, oriented along the edge dislocation lines of the 12 glide systems. The detection of a single $\langle 112\rangle$ rotation axis is interpreted as the presence of a number of single-orientation unpaired-edge GNDs in the probe volume. A local accumulation of GNDs typically arises near a grain boundary or a neutral fiber, against which dislocations pileup (leading to elongated spots), or in strongly distorted regions in which dislocation walls separate relatively undistorted crystal blocks (leading to split spots).

Several hypotheses are used in the description below. The local orientation gradient, of interest here, is a $3 \times 3$ matrix, obtained from the initially $9 \times 3$ gradient by neglecting the elastic-strain gradient (spatial variations of the shape of the unit cell). Two further simplifications are made: (i) the orientation gradient is constant over the probe volume, and along a single direction, so that a vector can be used to represent it; (ii) the orientation spread is around a single-rotation axis $\mathbf{v}$. The gradient vector is then simply $\operatorname{grad}\left(\theta_{\mathrm{v}}\right)$ or $\nabla\left(\theta_{\mathrm{v}}\right)$, where $\theta_{\mathrm{v}}$ is the rotation angle around $\mathbf{v}$. A gradient perpendicular to the rotation axis (as in the case of edge dislocations) gives a fan-like orientation (Fig. 5.A.1a). A gradient parallel to the rotation axis gives a screw-like orientation (Fig. 5.A.1b).

The elongation $\Delta \mathbf{q}$ (vector) of a spot with diffraction vector, $\mathbf{q}$, is the product of two terms:

\section{A first term:}

$$
\|\mathbf{q}\| \cdot|\sin \alpha| \cdot(\mathbf{v} \times \mathbf{q}) /\|\mathbf{v} \times \mathbf{q}\|
$$

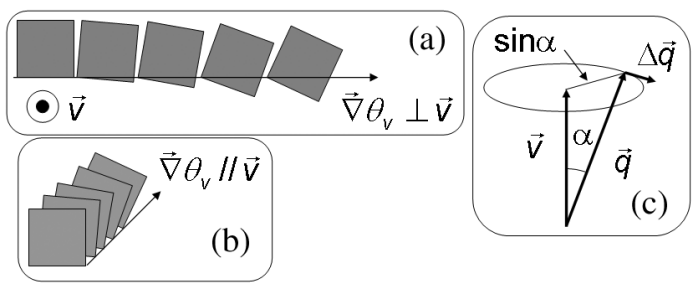

Fig. 5.A.1. (a, b) Illustration of lattice rotation gradient with the rotation axis $\mathbf{v}$ perpendicular (a) or parallel (b) to the gradient $\nabla\left(\theta_{\mathrm{V}}\right)$. (c) Rotation of reciprocal space vector, $\mathbf{q}$, around real space axis, $\mathbf{v}$. 
that gives the direction of the spot elongation, and the dependence of its amplitude on $\mathbf{q}$ (i.e. on the spot). This term expresses the rotation of $\mathbf{q}$ around $\mathbf{v}$ with a $(\mathbf{q}, \mathbf{v})$ angle equals to $\alpha$ (Fig. 5.A.1c). It is maximum for the spots with $\alpha=90^{\circ}$ and zero for $\alpha=0 . \Delta \mathbf{q}$ is in reciprocal space and needs to be converted to detector space for practical use.

2. A second term, $\Delta \theta_{\mathrm{v}}$, that expresses the orientation variation of the crystal lattice between the two ends of the probe volume, along the direction of the rotation gradient:

$$
\Delta \theta_{\mathrm{v}}=\sqrt{\left(\left(\mathrm{d} \theta_{\mathrm{v}} / \mathrm{dx} \cdot \mathrm{sx}\right)^{2}+\left(\mathrm{d} \theta_{\mathrm{v}} / \mathrm{dy} \cdot \mathrm{sy}\right)^{2}+\left(\mathrm{d} \theta_{\mathrm{v}} / \mathrm{dz} \cdot \mathrm{sz}\right)^{2}\right)}
$$

where $\mathbf{s}=(\mathbf{s x}, \mathbf{s y}, \mathbf{s z})$ (three perpendicular vectors) describes the approximate shape of the probe volume (Fig. 5.A.2c). This term expresses the proportionality of the elongation with respect to the gradient, and with respect to the length of the probe volume along the gradient.

For edge dislocations:

$$
\operatorname{grad}\left(\theta_{\mathrm{v}}\right)=\rho_{\mathrm{GND}} \cdot \mathbf{b}
$$

where $\rho_{\mathrm{GND}}$ is the local density of unpaired edge dislocation lines (per unit area perpendicular to the lines), and $\mathbf{b}$ is the Burgers vector.
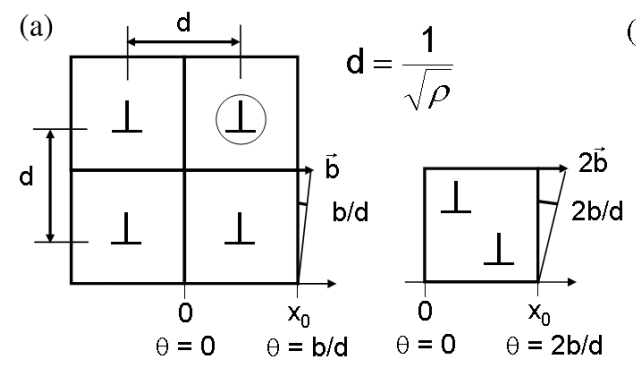

(b)

(c)
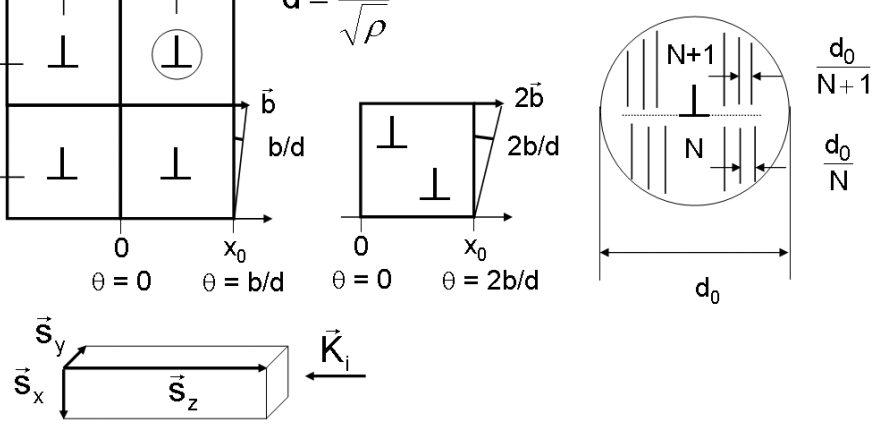

Fig. 5.A.2. (a) Illustration of Eq. (5.4) that links the length of the probe volume along $\mathbf{b}$, the GND density $\rho_{\mathrm{GND}}$, and the length of the Burgers vector, to the variation of angle $\Delta \theta_{\mathrm{V}}$ between the two ends of the probe volume. (b) Rough explanation of the dominant strain term (radial dq/q) due to an edge dislocation. (c) Typical probe volume $\mathrm{s}=(\mathbf{s x}, \mathbf{s y}, \mathbf{s z})$ for the $2 \mathrm{D}$ version of the technique. The definition of the $\mathrm{x}, \mathrm{y}, \mathrm{z}$ frame is local to this section, see Fig. 5.B.1 for the main definition. $\mathbf{K}_{\mathbf{i}}$ is the direction of the incident beam. 
The second term then becomes:

$$
\Delta \theta_{\mathrm{v}}=\rho_{\mathrm{GND}} \cdot \mathbf{b} \cdot \mathrm{L}_{-} \mathbf{b}
$$

where $L \_b$ is the length of the probe volume along $\mathbf{b}$.

$$
L_{-} b=\sqrt{\left(\left(\mathbf{u}_{\mathbf{b}} \cdot \mathbf{s x}\right)^{2}+\left(\mathbf{u}_{\mathbf{b}} \cdot \mathbf{s y}\right)^{2}+\left(\mathbf{u}_{\mathbf{b}} \cdot \mathbf{s z}\right)^{2}\right)}
$$

where $\mathbf{u}_{\mathbf{b}}$ is the unit vector along $\mathbf{b}$.

The product of (5.1) and (5.4) then leads to the well-known formula for $\Delta \mathbf{q}$ given, for example, in Ice and Barabash (2007). Figure 5.A.2 a provides an illustration of Eq. (5.4). The probe volume is between $\mathbf{x}=0$ and $\mathbf{x}=\mathbf{x} 0$, with $\mathbf{x}$ parallel to $\mathbf{b}$. The angle variation $\Delta \theta_{\mathrm{v}}$ between $\mathbf{x}=0$ and $\mathbf{x}=\mathbf{x} 0$ is given by the number $\mathrm{n}_{\mathrm{GND}}$ of dislocations between $\mathbf{x}=0$ and $\mathbf{x}=\mathbf{x} 0$, multiplied by $\mathbf{b} / \mathbf{d}$, where $\mathrm{d}$ is the average distance between the dislocations along $\mathrm{y}$. Equation (5.4) then follows since $\rho_{\mathrm{GND}}=n_{\mathrm{GND}} /(\mathrm{x} 0 \cdot \mathrm{d})$ and $\mathrm{L} \_\mathrm{b}=\mathbf{x} 0$.

In practice, for an $\mathrm{FCC}$ lattice, if the experimental $\mathbf{v}$ axis coincides with a $\langle 112\rangle$ direction, the hypothesis of single-orientation GNDs is reasonable, and the table of the 12 possible glide systems provides $\mathbf{b}$ (knowing $\mathbf{v}$ ). Knowing the probe volume and the mean orientation of the crystal with respect to the probe volume, the knowledge of $\mathbf{b}$ allows the calculation of $L_{-} b$, and the conversion of the measured spot FWHM into $\rho_{\mathrm{GND}}$. The consistency with the hypothesis of single-slip and pure-edge dislocations can then be checked by examining whether all the spots lead to the same $\rho_{\mathrm{GND}}$.

\section{A.3. Orientation gradient (GNDs): two-point measurements}

When a single non- $\langle 112\rangle$ rotation axis is present, whose orientation was identified using the elongation directions, the FWHM of the spots provides the integral of the gradient on the probe volume, but obviously not the gradient.

In this case, and also in the case of a mix of axes (several elongation directions) the only way to find the direction of the gradient (assuming that the gradient can be described by a single vector) is to probe another point of the sample. A first possibility is to examine how the spots move on the detector, i.e. how the mean lattice (obtained by refining the orientation matrix) rotates (and deforms), when the measurement point moves on the 
sample. A second possibility is to examine how the spot width increases when multiplying by two the X-ray beamsize along $\mathbf{x}$ or $\mathbf{y}$.

The method in which the probe volume is displaced is used in electron back-scatter diffraction (EBSD) (cf. Chapter 12; Pantleon, 2008; Kysar et al., 2010) and also Laue microdiffraction (Larson et al., 2007) to extract GND densities on the various glide systems in the multi-slip case. To mathematical solve the problem, however, requires additional hypotheses (e.g. minimization of total dislocation line length), as there are too many unknown densities, even when measuring the nine components of the orientation gradient (which requires 3D mapping).

\section{A.4. Influence of the probing depth}

In the $2 \mathrm{D}$ version of the technique the probe volume is elongated along the incident beam (cylinder). Taking $\mathrm{z}$ along the beam and $\mathbf{x}, \mathbf{y}$ perpendicular to the beam (temporarily), sz is much larger than sx and sy (typically 5 to 50 times for copper, depending on spot energy). The FWHM therefore shows a disproportionate sensitivity to the $\mathbf{z}$ component of the orientation gradient (with respect to the $\mathbf{x}$ and $\mathbf{y}$ components). In the case of multiple slip with edge dislocations on several systems and (correspondingly) multiple elongation directions, the most visible system will be the one with $\mathbf{b}$ closest to the incident beam direction. The probe depth sz, which is necessary for the calculation of L_b, can usually be calculated using the material X-ray absorption and the spot energy. The approximate spot energy is obtained from the analysis of the Laue pattern (assuming zero hydrostatic strain). If the crystal is still perfect enough for dynamical diffraction effects to occur, the probe depth is given by the (much shorter) extinction length.

The interest of the 3D mode (spatial resolution along the incident beam) then becomes clear: for single-point FWHM measurements, this mode provides a more cubic probe volume. For the two-point orientation gradient measurements, it allows the extraction of the $\mathbf{z}$ component of the gradient.

\section{A.5. Screw components}

Screw components of dislocations are often neglected in the attempts to extract GND densities, as the lattice rotations they produce are smaller 
than the ones due to the edge dislocations (i.e. they may be masked by edge components). The corresponding rotations' fields are also more complex, the simplest field corresponding to a precession of the lattice.

Screw dislocations can be detected when there is a very high screw/edge proportion in the probe volume. A calculation by Wilson (1952) predicts a ring shape for the spot with $\mathbf{q}$ along $\mathbf{b}$, assuming a small cylindrical probe volume around the dislocation line.

\section{A.6. Strain spread}

The white-beam method, based on orientation spreads, essentially only detects GNDs. The transverse width (dq perpendicular to $\mathbf{q}$ ) of the spots is also sensitive to paired dislocations (SSDs) but with a lower exponent with respect to $\rho$ ( $\sqrt{\rho}$ instead of $\rho)$, due to the screening of the orientation/strain fields between closely spaced dislocations of opposite signs. Also, the spread due to SSDs does not show the proportionality to the length of the probe volume present for GNDs. For fixed densities $\rho_{\mathrm{GND}}$ and $\rho_{\mathrm{SSD}}$, if the probe volume is enlarged along $\mathbf{b}$, the GND contribution increases but the SSD contribution stays constant. A small number of GNDs is therefore sufficient to mask a large number of SSDs. The sensitivity to SSDs is typically 50 times smaller than for GNDs. ${ }^{2}$

The total dislocation density is therefore difficult to obtain from the transverse width of the Laue spots: SSDs and GNDs contribute with widely different weights, and supplementary information on their respective proportions would be needed. This density can however be obtained by measuring the radial width (dq parallel to q), since GNDs and SSDs contribute with similar weights to this width. The only way to measure the radial width without rotating the sample is to measure the energy width of the Laue spot. This can be performed by monochromatizing the incident beam and scanning its energy.

\footnotetext{
${ }^{2}$ Assuming the experimental setup at BM32 with a sample-detector distance of $\sim 70 \mathrm{~mm}$ and the MARCCD detector, a transverse FWHM dq/q of $1 \mathrm{mrad}$ (which corresponds to 1 (2) pixels for a spot at the center of the detector from $\chi(2 \theta)$ elongations) corresponds to $\rho_{\mathrm{SSD}}=1.9 .10^{14} / \mathrm{m}^{2}=190 / \mu \mathrm{m}^{2}$ and $\rho_{\mathrm{GND}}=4.10^{12} / \mathrm{m}^{2}=4 / \mu \mathrm{m}^{2}$ (for a probe volume of $1 \mu \mathrm{m}$ along $\mathbf{b}$, with $\mathbf{b}=2.5 .10^{-10} \mathrm{~m}$ for copper).
} 
Energy scans allow a good reciprocal space resolution $\left(\mathbf{d q} / \mathbf{q}=1.10^{-4}\right.$ instead of $1.10^{-3}$ per pixel using white beam), better adapted to measure small widths.

Calculations (e.g. Krivoglaz, 1995) show that the (comparatively small) radial width behaves as $\sqrt{\rho}$, where $\rho$ is the total dislocation density. ${ }^{3}$ This width arises from the elastic strain field of the dislocations. For an edge dislocation, the leading term of this strain field can be visualized as follows: the distance between atomic planes perpendicular to $\mathbf{b}$ is slightly compressed above the glide plane (in the half-space with the additional atomic plane) and slightly expanded below (Fig. 5.A.2b), hence a spread in d-spacing for diffraction vectors along $\mathbf{b}$.

For an ideal experiment, it would therefore be desirable to couple whitebeam and monochromatic beam-measurements for a complete picture of the spatial distribution of dislocations.

\section{Appendix 5.B. The Compressive/Tensile Test with Single Slip: An Attempt to Predict Lattice Rotation Fields}

\section{B.1. Introduction}

Figure 5.7 (in Section 5.4) gathers in a stereographic projection all the available knowledge in terms of crystalline orientation of the various geometrical elements related to a given pillar (e.g. cutting faces) installed in a given testing device (e.g. compressive axis), in a given microdiffraction setup (e.g. incident beam, detector). The goal of this appendix is to explain the interest of such a figure (in particular the role of the $\mathbf{z}_{1} \_\mathbf{b}$ and $\mathbf{z}_{\mathbf{1}} \mathbf{n}$ axes), and the interest of using it to draw the glide traces of system 1 on the pillar faces, for comparison with the SEM images taken after the test. The square section of the pillars is useful here as it helps to link the diffraction and the SEM.

The situation of single slip is, in theory, the simplest one, when trying to model the evolution of a population of dislocations, in a material submitted to a uniaxial shear stress. This theoretical simplicity turns to high complexity in practice, as real data are collected with tensile or compressive tests.

\footnotetext{
${ }^{3}$ Note that the GND/SSD character is very local and scale-dependent: a given dislocation can end up as a SSD or a GND depending on the size and/or position of the probe volume.
} 
The goal of the mechanical tests described in Section 5.4 is to approach, with a tensile or compressive test on a single-crystalline pillar, a shear experiment with single slip on system 1 (i.e. with only one slip direction $\mathbf{b}_{\mathbf{1}}$ and one glide plane $\mathrm{P}_{1}$ ), in which imposed displacements would directly be applied to two $\mathrm{P}_{1}$ planes limiting the sheared region.

The force applied in practice is uniaxial, tensile or compressive: it can be attempted to concentrate it into a resolved shear stress on system 1, but a parasitic fraction always remains on the other systems (in particular a nonnegligible fraction on system 2 , which also has $\mathrm{P}_{1}$ as glide plane). System 1 needs to dominate not only at the beginning of the test, but also throughout the test, after possible lattice rotations.

This optimization of the transmission of the uniaxial stress toward shear on system 1 (or more precisely the differential transmission between systems 1 and 2) leads to a cutting of the pillar along low-symmetry axes of the crystal. Due to this cutting, the slip plane and the slip directions have complex orientations - in theory, the most favorable $\mathbf{x}$ loading axis is a [538] crystal direction. This gives a $\mathrm{P}_{1}$ plane at $35.7^{\circ}$ from $\mathbf{x}$, with an angle between $\mathbf{x} \times \mathbf{n}_{\mathbf{1}}$ and $\mathbf{x} \times \mathbf{b}_{\mathbf{1}}$ of $24.2^{\circ}$ (where $\mathbf{n}_{\mathbf{1}}$ is the normal to $\mathrm{P}_{1}$ ). The slip direction therefore strongly deviates from the line of greatest slope ( $\mathrm{x}$-wise) of the $\mathrm{P}_{1}$ plane (Fig. 5.B.1a).

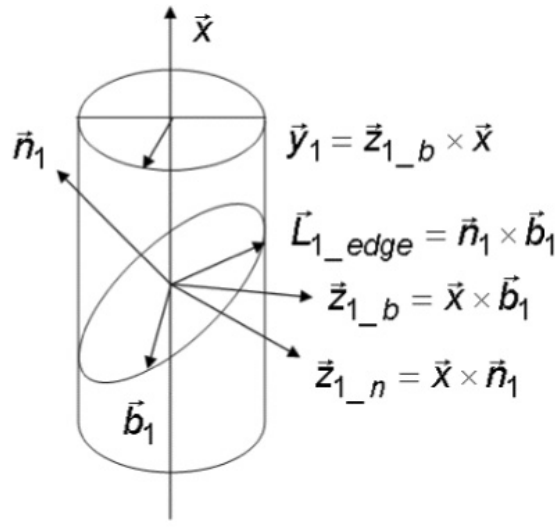

(a)

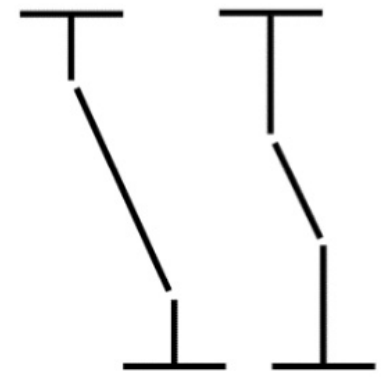

(b)

Fig. 5.B.1. (a) Directions of $\mathbf{b}_{\mathbf{1}}, \mathbf{n}_{\mathbf{1}}$ and $\mathrm{P}_{1}$ for a pillar cut along the [538] axis. (b) Example of zigzag shape arising from pillar segments with different amounts of shear. 
Single-slip shear, when applied to a segment of the pillar, translates the top end of the segment along $\mathbf{b}_{\mathbf{1}}$, with respect to the bottom end.

For a pillar initially oriented along $\mathrm{x}$, this displacement can be decomposed into a part along $\mathrm{x}$, and a part along $\mathbf{y}_{\mathbf{1}}=\mathbf{x} \times\left(\mathbf{b}_{\mathbf{1}} \times \mathbf{x}\right)$. For a segment with homogeneous shear, this leads to a rotation of the axis of the segment around $\mathbf{z 1} \_\mathbf{b}=\mathbf{x} \times \mathbf{b}_{\mathbf{1}}$ (note that the microscopic material rotation, obtained after removing the shape of the sheared volume is around $\mathbf{n}_{\mathbf{1}} \times \mathbf{b}_{\mathbf{1}}$ and not $\left.* *\right)$.

As the shear proceeds, the external shape of the crystal therefore loses its symmetry, which causes the appearance of parasitic bending and torsion torques. These torques produce local deviations with respect to the theoretical uniaxial stress, and may increase the Schmid factors of secondary systems, and activate some of these systems.

One interesting characteristic of Fig. 5.7 is to offer a visual comparison between the expected bending/torsion axes, and the $\mathbf{L}_{\mathbf{e d g e}}=\mathbf{b} \times \mathbf{n}$ rotation axes, and the $\mathbf{n}$ normals to the glide planes, for the various slip systems. This allows a fast analysis of the potentially activated systems, typically the ones with $\mathbf{L}_{\mathbf{e d g e}}$ nearly parallel to the axis for a bending torque, and the ones with $\mathbf{n}$ nearly parallel to the axis for a torsion torque.

One source of torques is the non-uniformity in the amount of shear. This non-uniformity leads to differently oriented material segments (zigzag shape). The force applied along $\mathbf{x}$ to the ends of the pillar is then transmitted as bending torques at the junctions between the segments. For tensile tests, the applied force will tend to reduce the amplitude of the zigzag, while for compressive tests, it will tend to increase its amplitude. An unavoidable non-uniformity is the one occurring between the heads (with zero shear) and the middle part (with significant shear) of the pillar, due to the difference of thickness between the two (Fig. 5.B.1b).

In sections 5.B.2 and 5.B.3 the following questions will be addressed:

- What could be the definition of the best-behaved test, given the symmetry incompatibilities between the ideal unaxial compressive/tensile test and the ideal single-slip test (Section 5.B.2)?

- Which bending and torsion axes will appear during the test, given various situations for the boundary conditions at the ends (Section 5.B.3)? Here the loading device is supposed to be initially well aligned with respect to the pillar. 
In these two sections, it will be assumed that: (i) only system 1 is activated, and (ii) the dislocations created on system 1 escape at the surface (or at least the ones contained in $\mathrm{P}_{1}$ planes that only cross the lateral faces of the pillar), without storage. The next section will examine how to test hypothesis (ii) experimentally.

Indeed, a recurring question in plasticity tests on FIB-cut micropillars is the influence of the near-surface structure of the lateral faces. The image force attracts the dislocations to the surfaces but, in order for them to escape (as steps for edge dislocations, and as surface shear for screw dislocations), the crystal's near-surface region needs to show a good crystalline quality. Otherwise it may act as a barrier, against which dislocations will pileup. This pileup may in turn change the distribution of stresses in the pillar's section, by concentrating them close to the lateral faces.

Section 5.B.4 will examine how to combine:

- The information contained in the SEM images taken after the test.

- The knowledge of the macroscopic orientation of the pillar's faces during the Laue microdiffraction measurements.

This examination should determine:

- Where in the Laue maps (measured after unloading) the incident X-ray beam cross regions will be likely to show surface storage.

- If the possibility exists to detect dynamical surface storage while keeping the X-ray beam at the center of the pillar (sample holder motions being forbidden during the test).

In this appendix, only the effects detectable by the white-beam method are of interest, i.e. only lattice rotations. Dislocations appearing during the test in the form of loops will be mostly invisible here, as long as the loop size is smaller than $1 \mu \mathrm{m}$. The accumulation of SSDs on system 1, which is the main change in the dislocation population expected for homogeneous single slip with anchoring points in the volume, is therefore almost impossible to detect here.

\section{B.2. Best compromise for a single-slip tensile/compressive test}

A really ideal tensile or compressive test, on a single crystal, is obtained when the following conditions are fulfilled (Fig. 5.B.2a): (i) two perpendicular planes $\mathrm{Py}$ and $\mathrm{Pz}$ that contain the load axis $\mathbf{x}$ are mirror-symmetry 


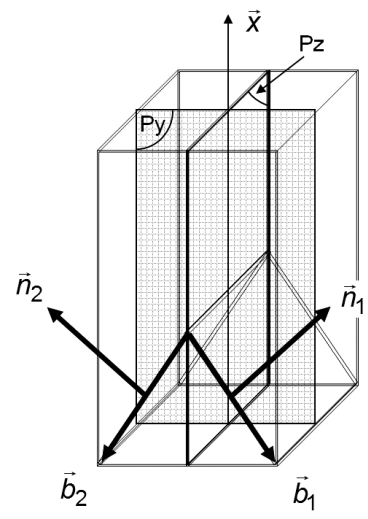

(a)

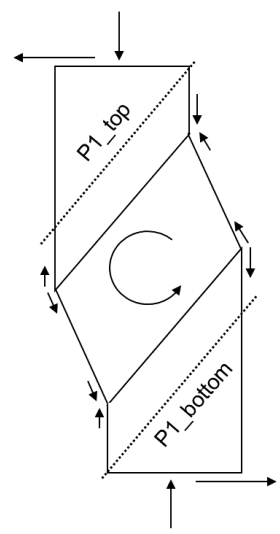

(b)

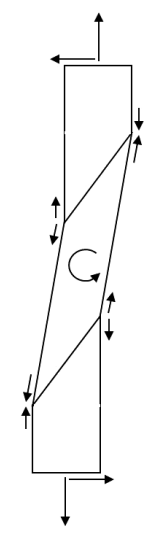

(c)

Fig. 5.B.2. (a) The ideal tensile/compressive test on a single crystal: double slip with two mirror symmetries. (b, c) Best compromise for a tensile $\mathrm{T} 1$ (c) or compressive $\mathrm{C} 1$ (b) test producing single slip.

planes of the crystal, and (ii) the symmetry of the problem with respect to these two planes is conserved throughout the whole test. To obtain these symmetry features, a configuration of double slip is necessary, with two $\mathrm{P}_{1}$ and $\mathrm{P}_{2}$ slip planes that are mirrors images of each other with respect to $\mathrm{Py}$, glide directions $\mathbf{b}_{1}$ and $\mathbf{b}_{2}$ parallel to $\mathrm{Pz}$, and, during the test, a permanent balancing between the levels of shear on the two systems. The single-slip test of interest here clearly does not fulfill these conditions: the crystal may present a symmetry plane containing $\mathrm{x}$ in the initial state, but this symmetry plane will not be preserved during the test, as $\mathbf{b}_{\mathbf{1}}$ deviates by several tens of degrees from the line of greatest slope of $\mathrm{P}_{1}$.

An ideal single-slip test would consist of cutting a crystal slice with two faces parallel to $P_{1}$, and in imposing a translation along $\mathbf{b}_{1}$ of one face with respect to the other. The cutting along $\mathrm{P}_{1}$ provides faces that keep the same crystallographic orientation during the test. Any other orientation of the faces would require a lattice rotation to maintain the faces parallel to a given plane of the laboratory frame during the shear (Reid, 1973). For homogeneous shear between two faces of normal $\mathbf{A}$, calculations predict a lattice rotation around $\mathbf{A} \times \mathbf{n}_{\mathbf{1}}$.

The best compromise between the two tests consists in a slicing of the pillar into three segments, separated by interfaces parallel to $\mathrm{P}_{1}$ (Fig. 5.B.2b): a central segment with homogeneous non-zero shear, and 
two end segments with zero shear. The end segments therefore act as prolongations of the ends, with pushing surfaces parallel to $\mathrm{P}_{1}$.

The central segment may potentially extend up to the $P_{1 \_t o p}$ and

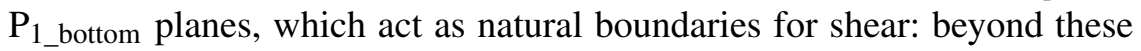
planes, the progress of edge dislocations of one of the two signs is blocked by a barrier (low-stress region in a thick part, or imposed-displacement surface against the punch). The resulting dislocation pileup creates a backstress that counteracts the applied stress, and slows down the slip in this region.

The question is then: in practice, which boundary conditions at the ends of the pillar would be the best to favor such a segmentation of the pillar into sections limited by planes parallel to $\mathrm{P}_{1}$ ? For the compressive test, a fixed orientation of the heads, combined with a possibility of lateral displacement of the punch $\left(\mathrm{C}_{1}\right.$ case $)$, may be adequate. The crystal then guides the motion along $\mathbf{b}_{1}$, deviating the force from the compression axis and shifting the punch off-axis. For the tensile test, this combination of boundary conditions is unfavorable due to the natural realignment of the mean pillar axis on the applied force, which maintains the grip in its center position. The best case would correspond to free rotation of the ends around all axes ( $\mathrm{T}_{1}$ case).

\section{B.3. Lattice rotations, bending and torsion axes}

\section{B.3.1. Best-compromise case}

For the $\mathrm{C}_{1}$ case, lattice rotation is zero for the pillar's end segments, except for the small storage on system 1 , which leads to a rotation around $\mathbf{L}_{\mathbf{1} \_ \text {edge }}=$ $\mathbf{n}_{\mathbf{1}} \times \mathbf{b}_{\mathbf{1}}$. Lattice rotation is also zero for the central segment, except for the small increase of the zigzag caused by the applied force that adds a rotation around $\mathbf{z}_{1 \_} \mathbf{b}$.

For the $T_{1}$ case, the same effects are expected, but superimposed to a global rotation of the pillar (ends included) around $\mathbf{z}_{\mathbf{1} \_\mathbf{b}}$, due to the realignment of the mean pillar axis on the applied force. In addition, the small rotation coming from the zigzag is reverted, as the applied force tends to unbend the zigzag (Fig. 5.B.2b).

The zigzag aspect leads to an additional bending torque around $\mathbf{z}_{1} \_\mathbf{b}$ at the interfaces between the segments. For $\mathrm{C}_{1}$, the convex parts will be under tension (i.e. reducing the applied compressive stress) and the concave parts 
under compression. For $\mathrm{T}_{1}$, the convex parts will be under compression (i.e. reducing the applied tensile stress) and the concave parts under tension.

\section{B.3.2. Case of real boundary conditions}

The real compressive and tensile tests are designed in such a way that, (i) the orientation of the ends is fixed, and (ii) the base is fixed, and the lateral motions of the punch/grip are forbidden.

For compressive tests, however, case $\mathrm{C}_{1}$ is observed on a small strain range, i.e. condition (ii) is relaxed. This is attributed to the existence of a lateral play of the order of $1 \mu \mathrm{m}$ between the punch holding rod and its guiding rail, combined to a certain flexibility of the punch's rod. Additional analyses would be needed to elucidate if the play and the lateral spring force of the punch rod show an anisotropy in the yz plane. A deviation from the expected real boundary conditions therefore improves the ideality of the test. In what follows, condition (ii) is supposed to hold.

In practice, an important experimental parameter is the orientation of the Pa interfaces (here assumed to be flat) between the zero-shear segments (heads) and the non-zero-shear segment (center) of the pillar. Indeed, real tests reveal that these $\mathrm{Pa}$ interfaces (supposed to be planar) are unfortunately not always parallel to $\mathrm{P}_{1}$. Assuming uniform shear in the central segment, a lattice rotation around $\mathbf{A} \times \mathbf{n}_{\mathbf{1}}$ is expected in this segment, where $\mathbf{A}$ is the normal to $\mathrm{Pa}$. The associated creation of a flat tilt boundary along $\mathrm{Pa}$ requires an ordering of the shear-induced dislocations.

The most classic case is with $\mathbf{A}$ along $\mathbf{x}$, giving the $\mathrm{C}_{2}$ and $\mathrm{T}_{2}$ cases for compressive and tensile tests, respectively. The rotation axis then becomes $\mathbf{z}_{1 \_\mathbf{n}}=\mathbf{x} \times \mathbf{n}_{\mathbf{1}}$.

In the $\mathrm{C} 2$ case, the whole pillar undergoes a shear up to the top surface and down to the base. The argument of confinement of the sheared region between the $\mathrm{P}_{1 \_ \text {top }}$ and $\mathrm{P}_{1 \_ \text {bottom }}$ does not hold anymore. This spread of the shear over the larger height of the pillar may lead to less visible effects in the SEM in terms of glide traces, compared to case $C_{1}$. Reid (1973) also mentions a non-uniformity of the resolved shear stress in the $\mathrm{P}_{1}$ plane due to the inclined character of the pillar.

In the $T_{2}$ case, the $\mathbf{z}_{1} \_\mathbf{n}$ rotation of the central part would in theory be superimposed to the global rotation (frustrated to zero for the ends) around $\mathbf{z}_{1 \_} \mathbf{b}$ imposed by the realignment of the pillar on the force. In practice, 
conditions (i) and (ii) applied to tensile pillars with a large aspect ratio seem to favor a multisegment zigzag structure, with uniform shear over only very short segments. The $\mathbf{z}_{1 \_\mathbf{b}}$ rotation in the central part appears to dominate over the $\mathbf{z}_{1} \_\mathbf{n}$ rotation.

The square section of the pillar may bring an additional torque around $\mathbf{x}$, as a secondary effect of an elastic bending of the pillar around an axis perpendicular to $\mathbf{x}$ (e.g. $\mathbf{z}_{1} \_\mathbf{n}$ or $\mathbf{z}_{\mathbf{1} \_\mathbf{b}}$ ), and not parallel to one of the lateral faces. This twist would arise because a bent square-section beam tries to recover its natural bending axes that are parallel to the lateral faces.

\section{B.4. GND storage against the pillar's lateral faces}

In real experiments, a certain resistance of the free surface might exist with respect to the emergence of loops and spiral single arms. This resistance might stem from either a native oxide layer or an FIB damage layer. An intermediate stage is therefore expected in the multiplication-escape dislocation mechanism, in which dislocation lines pileup against the lateral faces (in the $\mathrm{P}_{1}$ planes). Several rotations of the source may be necessary to overcome the critical shear for emergence, but even for high surface resistance, a few piled up dislocations should be sufficient to make the head dislocation pierce the barrier - at the head of the pileup, the macroscopic applied stress is multiplied by the number of piled up dislocations.

For a square-section pillar, a question may then arise: will the critical shear for emergence depend on the edge/screw character of the dislocation line piled against the surface? If yes, unescaped fragments of the loops may remain in the crystal, anchored to the surface at both ends.

Another question is: where to look for GNDs stored against the damaged/oxidized surface, when analyzing Laue maps? When such storage is present, the pillar can approximately be described as a core-shell structure, with a high-GND-density shell (with GNDs of opposite signs on the opposite faces of the pillar), and a low-GND-density core. An SEM image with glide traces (e.g. Fig. 5.B.3e) is then needed, in order to locate the pillar segments where single slip is strongly activated. Indeed, the shell is only expected around these segments. For example, in Fig. 5.B.3e, the activated segment is around $3 \mu \mathrm{m}$ high (along $\mathbf{x}$ ) in the $7 \times 7 \times 21 \mu \mathrm{m}^{3}$ pillar. 


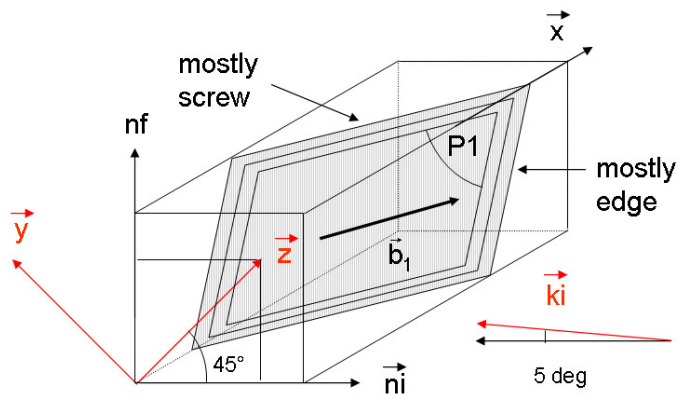

(a)

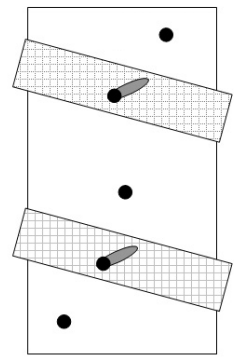

(c)

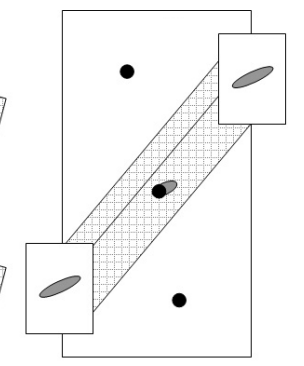

(d)

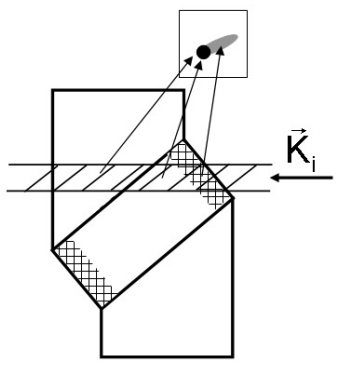

(b)

Fig. 5.B.3. (a) Typical geometry for a compression pillar, with the $\mathrm{P}_{1}$ slip plane and $\mathbf{b}_{\mathbf{1}}$ slip direction, and the incident beam direction $\mathbf{k}_{\mathbf{i}}$. The dislocations on system 1 piled up against the lateral faces are mostly edge on the i face and mostly screw on the f face. (b) Expected spot shape when the incident X-ray beam crosses the shell on a face whose piled up dislocations are mostly edge. (c) Expected spot shapes in Laue map for pillar oriented as in (a) or (b). (d) Same as (c) after a rotation of the pillar by $90^{\circ}$ around its axis, (e) An SEM image of a deformed pillar (from Kirchlechner et al., 2011a).

A variation of orientation of the unit cell is expected between the core and the shell of the pillar: slow in the core and fast in the shell. This variation is expected to take place along $\mathbf{b}_{\mathbf{1}}$, with a rotation axis $\mathbf{L}_{\mathbf{1} \_ \text {edge }}$ (taking into account only the edge dislocations). The orientation profile along $\mathbf{b}_{\mathbf{1}}$ should show a convex shape, as opposite sides of a loop produce rotations of the same sign with respect to the middle of the loop.

The best sensitivity to storage will be obtained when the X-ray beam crosses the shell on a lateral face whose piled up dislocations have a strong edge character.

In the experiments of Section 5.4, the incident beam is almost perpendicular to the $\mathrm{i}$ face, and almost parallel to the $\mathrm{f}$ face. From the 
crystallographic orientations of the $\mathrm{i}$ and $\mathrm{f}$ faces, the edge fraction $\mathrm{f}_{\text {_edge }}$ of the 1 dislocations piled against these two faces can be determined. This is done by calculating the angle between $\mathbf{L}_{\mathbf{1} \_ \text {edge }}$ and the glide trace (intersection between $\mathrm{P}_{1}$ and the face).

If the $i$ face has the largest $f_{\text {_edge }}$, the shell will be detectable with the beam at the center (laterally) of the pillar. The superposition of a sharp intense spot (pillar's core) and a broad low-intensity streak (shell), shifted with respect to the main spot, is then expected. If the f face has the largest $\mathrm{f}_{\text {_edge }}$, the shell will be detectable by placing the beam alternatively at the center and at the edge of the pillar (laterally). This should give the same two contributions as before, but separately (Fig. 5.B.3c,d).

\section{References}

Baudoing-Savois, R., De Santis, M., Saint-Lager M.-C., Dolle, P., Geaymond, O., Taunier, P., Jeantet, P., Roux, J.-P., Renaud, G., Barbier, A., Robach, O., Ulrich, O., Mougin, A. and Bérard, G. (1999). A new UHV diffractometer for surface structure and real time molecular beam deposition studies with synchrotron radiations at ESRF, Nucl. Instrum. Methods, B149, 213.

Berveiller, S., Malard, B., Wright, J., Patoor, J. and Geandier, G. (2011). In situ synchrotron analysis of lattice rotations in individual grains during stressinduced martensitic transformations in a polycrystalline CuAlBe shape memory alloy, Acta Mater., 59, 3636-3645.

Bleuet, P., Gergaud, P., Micha, J.-S., Robach, O., Rieutord, F. (in preparation, 2013). In situ Laue microdiffraction study of electromigration in copper interconnects, Acta Mater.

Chung, J.-S. and Ice, G.E. (1999). Automated indexing for texture and strain measurement with broad-bandpass X-ray microbeams, J. Appl. Phys., 86, 5249-5255.

Csikor, F.F., Motz, C., Weygand, D., Zaiser, M. and Zapperi, S. (2007). Dislocation avalanches, strain bursts, and the problem of plastic forming at the micrometer scale, Science, 318, 251.

Daveau, G. (2012). Interaction dislocations - joints de grains en déformation plastique monotone: étude expérimentale et modélisations numériques, $\mathrm{PhD}$ Thesis, Ecole Centrale Paris.

Devincre, B., Hoc, T. and Kubin, L. (2008). Dislocation mean free paths and strain hardening of crystals, Science, 320, 1745-1748. 
Frank, F.C. and Read, W.T. (1950). Multiplication processes for slow moving dislocations, Phys. Rev., 79(4), 722.

Gatti, R. and Devincre, B. (in preparation, 2013). Simulation of X-ray diffraction peaks broadening in dislocated materials, J. Appl. Crystallogr.

Greer, J.R. and Nix, W.D. (2006). Nanoscale gold pillars strengthened through dislocation starvation, Phys. Rev. B, 73, 24, 245410.

Hignette, O., Cloetens, P., Rostaing, G., Bernard, P. and Morawe, C. (2005). Efficient sub $100 \mathrm{~nm}$ focusing of hard X-rays, Rev. Sci. Instrum., 76, 063709.

Hirth, J.P. and Lothe, J. (1968). Theory of Dislocations, New York: McGraw-Hill.

Hull, D. and Bacon, D.J. (2001). Introduction to Dislocations, Oxford: Butterworth-Heinemann.

Ice, G.E. and Barabash R.I. (2007). White beam microdiffraction and dislocations gradients, in F.R.N. Nabarro and J.P. Hirth (eds), Dislocations in Solids, Elsevier, Vol. 13, Chapter 79, 500-601.

Kaganer, V.M. and Sabelfeld, K.K. (2010). X-ray diffraction peaks from correlated dislocations: Monte Carlo study of dislocation screening, Acta. Crystallogr. A, 66, 703-716.

Kirchlechner, C., Imrich, P.J., Grosinger, W., Kapp, M.W., Keckes, J., Micha, J.-S., Ulrich, O., Thomas, O., Labat, S., Motz, C. and Dehm, G. (2012). Expected and unexpected plastic behavior at the micron scale: An in situ $\mu$ Laue tensile study, Acta Mater., 60(3), 1252-1258.

Kirchlechner, C., Keckes, J., Micha, J.-S. and Dehm, G. (2011c). In situ $\mu$ Laue: Instrumental setup for the deformation of micron sized sample, Adv. Eng. Mater., 13, 837.

Kirchlechner, C., Keckes, J., Motz, C., Grosinger, W., Kapp, M.W., Micha, J.-S., Ulrich, O. and Dehm, G. (2011a). Impact of instrumental constraints and imperfections on the dislocation structure in micron-sized $\mathrm{Cu}$ compression pillars, Acta Mater, 59, 5618.

Kirchlechner, C., Kiener, D., Motz, C., Labat, S., Vaxelaire, N., Perroud, O., Micha, J.-S., Ulrich, O., Thomas, O., Dehm, G. and Keckes, J. (2011b). Dislocation storage in single slip-oriented $\mathrm{Cu}$ micro-tensile samples: New insights via X-ray microdiffraction, Philos. Mag., 91, 1256.

Krivoglaz, M.A. (1995). X-Ray and Neutron Diffraction in Nonideal Crystals, Berlin: Springer.

Kysar, J.W., Saito, Y., Oztop, M.S., Lee, D. and Huh, W.T. (2010). Experimental lower bounds on geometrically necessary dislocation density, Int. J. Plasticity, 26, 1097-1123. 
Larson, B.C., El-Azab, A., Yang, W., Tischler, J.Z., Liu, W. and Ice, G.E. (2007). Experimental characterization of the mesoscale dislocation density tensor, Phil. Mag., 87, 1327-1347.

Larson, B.C., Yang, W., Ice, G.E., Budai, J.D. and Tischler, J.Z. (2002). Threedimensional X-ray structural microscopy with submicrometre resolution, Nature, 415, 887-890.

Maaß, R., Grolimund, D., Petegem, S.V., Willimann, M., Jensen, M., Van Swygenhoven, H., Lehnert, T., Gijs, M.A.M., Volkert, C.A., Lilleodden, E.T. and Schwaiger, R. (2006). Defect structure in micropillars using X-ray microdiffraction, Appl. Phys. Lett., 89, 151905.

Maaß R., Van Petegem, S., Van Swygenhoven, H., Derlet, P.M., Volkert, C.A. and Grolimund, D. (2007). Time-resolved Laue diffraction of deforming micropillars, Phys. Rev. Lett., 99, 145505.

Micha, J.-S. and Robach, O. (in preparation, 2013). Laue Tools: An open-source software for X-ray Laue microdiffraction analysis, J. Appl. Crystallogr.

Nix, W.D., Greer, J.R., Feng, G. and Lilleodden, E.T. (2007). Deformation at the nanometer and micrometer length scales: Effects of strain gradients and dislocation starvation, Thin Solid Films, 515, 3152.

Pantleon, W. (2008). Resolving the geometrically necessary dislocation content by conventional electron backscattering diffraction, Scripta Mater, 58, 994-997.

Petit, J., Bornert, M., Hofmann, F., Robach, O., Micha, J.-S., Ulrich, O., Le Bourlot, C., Faurie, D., Korsunsky, A.M. and Castelnau, O. (2011). Combining Laue microdiffraction and digital image correlation for improved stress field measurements with micrometer spatial resolution, Procedia IUTAM: Full Field Measurements and Identification in Solid Mechanics, 4, 133-143.

Reid, C.N. (1973). Deformation Geometry for Materials Scientists, International Series on Materials Science and Technology, Vol. 11, London: Pergamon Press.

Richard, A. (2012). Etude par diffraction des rayons X des déformations induites par irradiation / implantation d'ions dans le dioxyde d'uranium, PhDThesis, Université de Poitiers, France.

Richard, A., Palancher, H., Castelier, E., Micha, J.-S., Gamaleri, M., Carlot, G., Rouquette, H., Goudeau, P., Martin, G., Rieutord, F., Pirona, J.-P. and Garcia, P. (2012). Strains in light-ion-implanted polycrystals: Influence of grain orientation, J. Appl. Crystallogr., 45, 826-833.

Robach, O., Micha, J.-S., Ulrich, O. and Gergaud, P. (2011). Full local elastic strain tensor from Laue microdiffraction: Simultaneous Laue pattern and spot energy measurement, J. Appl. Crystallogr., 44, 688-696. 
Tamura, N., MacDowell, A.A., Spolenak, R., Valek, B.C., Bravman, J.C., Brown, W.L., Celestre, R.S., Padmore, H.A., Batterman, B.W. and Patel, J.R. (2003). Scanning X-ray microdiffraction with submicrometer white beam for strain/stress and orientation mapping in thin films, J. Synchrotron Radiat., 10, 137-143.

Uchic, M.D., Dimiduk, D.M., Florando, J.N. and Nix, W.D. (2004). Sample dimensions influence strength and crystal plasticity. Science, 305, 986.

Ulrich, O., Biquard, X., Bleuet, P., Geaymond, O., Gergaud, P., Micha, J.-S., Robach, O. and Rieutord, F. (2011). A new white beam X-ray microdiffraction setup on the BM32 beamline at the European Synchrotron Radiation Facility, Rev. Sci. Instrum., 82, 033908.

Ungar, T., Mugrahbi, H., Rönnpagel, D. and Wilkens, M. (1984). X-ray linebroadening study of the dislocation cell structure in deformed [001]-orientated copper single crystals, Acta Metall. Mater., 32(3), 333-342.

Villanova, J., Sicardy, O. Fortunier, R., Micha, J.-S. and Bleuet, P. (2010). Determination of global and local residual stresses in SOFC by X-ray diffraction, Nucl. Instrum. Meth. B, 268, 282-286.

Villanova, J., Sicardy, O., Fortunier, R., Micha, J.-S. and Bleuet, P. (2011). X-ray diffraction determination of macro and micro stresses in SOFC electrolyte and evolution with redox cycling of the anode, Mater. Sci. Forum, 681, 25-30.

Wilkens, M. and Bargouth, M.O. (1968). The determination of the dislocation density in deformed copper single crystals from X-ray diffraction profiles, Acta Metall. Mater., 16(3), 465.

Wilson, A.J.C. (1952). The diffraction of X-rays by distorted-crystal aggregates: IV. Diffraction by a crystal with an axial screw dislocation, Acta Crystallogr., 5, 318-322. 